\title{
The Dynamics of a Ringing Church Bell
}

\author{
J. Woodhouse, J. C. Rene, C. S. Hall, L. T. W. Smith, F. H. King, and J. W. McClenahan \\ Department of Engineering, University of Cambridge, Trumpington Street, Cambridge CB2 1PZ, UK
}

Correspondence should be addressed to J.Woodhouse, jw12@cam.ac.uk

Received 29 October 2012; Accepted 30 November 2012

Academic Editor: Emil Manoach

Copyright ( 2012 J. Woodhouse et al. This is an open access article distributed under the Creative Commons Attribution License, which permits unrestricted use, distribution, and reproduction in any medium, provided the original work is properly cited.

Church bell ringing as practised in the UK involves large-amplitude motions of both bell and clapper. A simulation model is developed and validated against experimental measurements. It is shown that the clapper does not hit the bell with a single impact but a long series of bounces, and this has important consequences for the decay profile of the bell vibration. Information relevant to bell-ringers and bell-hangers is collected in a series of design charts derived from the simulation model. These charts can assist in the diagnosis and correction of faults. Arising from the analysis of the bouncing clapper, a more general result is also presented relating to the frequency bandwidth when any structure is excited by a small bouncing impactor, for example an impulse hammer used in vibration testing.

\section{Introduction}

Church bells come under the general heading of tuned percussion instruments. A well-tuned bell gives a clear sense of a definite pitch when it is rung, because a few of the lower natural frequencies have been carefully adjusted to fall in harmonically related ratios (e.g., see Fletcher and Rossing [1]). However, the role of these natural frequencies in determining the sound of bells is not the topic of this paper. Instead, some less thoroughly explored dynamical aspects of the process of ringing church bells are investigated. The results have some relevance to the sound of bells, but at least as importantly, they shed light on how easily a ringer can control a bell, and how a bell-hanger might go about avoiding, or fixing, a problem. It should be emphasised from the start that this paper exclusively concerns bells rung "full circle," not bells that are "chimed" as in a carillon or a chiming clock.

Change-ringing on church bells, as practised mainly in the UK, involves the ringing of complex sequences of notes on bells that can weigh up to a tonne or more. The sequences are based on mathematical permutation algorithms called "methods" with strict rules, (e.g., see Wilson [2]). Bells suitable for change-ringing are suspended and rung in a way that makes them very unusual among percussion instruments.
Each bell is supported on a pivot so that, for each note struck, it can rotate through a full circle. The motion is controlled by the ringer's rope, which passes round a wheel fixed to the bell. The bell rotates in opposite directions for alternate strikes, called "handstroke" and "backstroke." The typical arrangements at the start of each are illustrated in Figure 1. The bell starts from an inverted position, the ringer pulls the rope, and the bell rotates through about $360^{\circ}$. At some time during the swing, the clapper strikes the bell. However, there is not a single, clean impact as one might expect on a xylophone or kettledrum, or indeed on a carillon bell. As will be shown by experimental results in the next section, the clapper bounces and undergoes a decreasing sequence of multiple impacts. Usually it has come to rest against the side of the bell by the time the swing is completed. Under normal circumstances, the ringer pulls the rope again more or less immediately to start the reverse swing and the next strike.

By making subtle changes in the amplitude of the swing near the top of the circle, just before or just after the moment when the swing stops and reverses, the ringer can make the necessary timing adjustments for controlling the position of the bell in the ringing sequence. As a result, the sound and "ringability" of bells depend not only on the linear acoustics of the modes and natural frequencies of the bells, but also on the rotational dynamics of the bell and clapper, interacting 


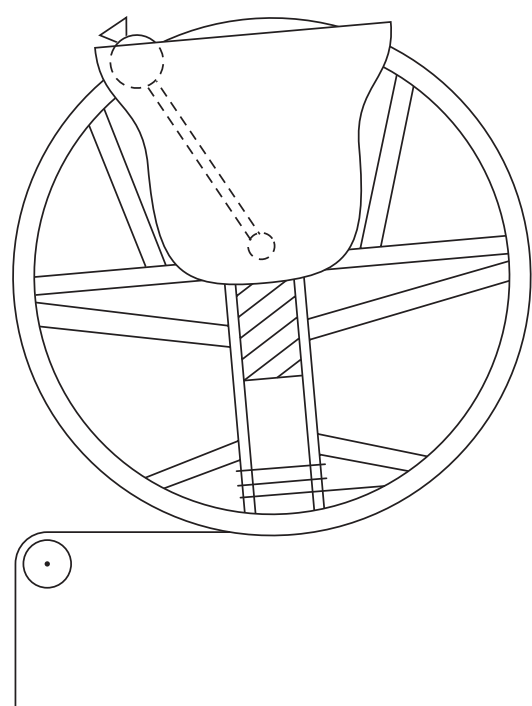

(a)

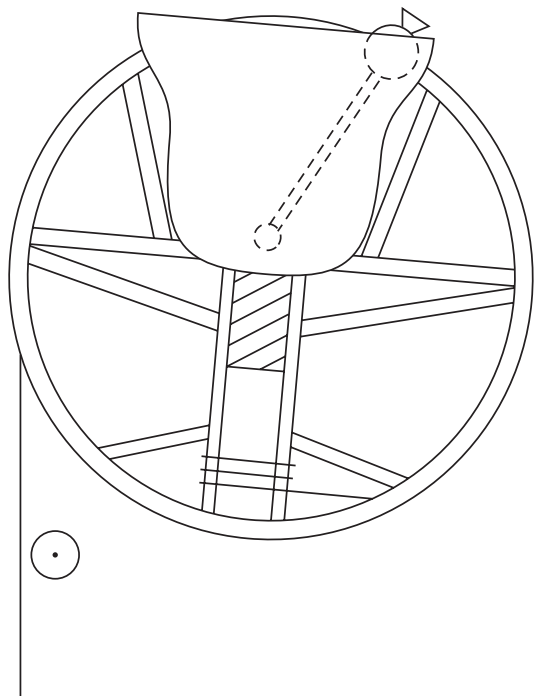

(b)

FIGURE 1: Sketches of a typical bell and wheel arrangement at (a) handstroke and (b) backstroke. Note the role of the pulley in allowing the ringer to initiate either stroke.

through impact each time the clapper strikes the bell surface. This is the subject of this paper. Experimental measurements on a relatively small bell in the laboratory, augmented by more limited tests on larger bells in a church tower, will be compared with the predictions of a numerical simulation.

The simulation program can be used for virtual design investigations into questions of importance to bell-hangers and ringers. Two quantities of interest are the bell angle at which the first clapper strike occurs and the velocity with which the clapper then strikes the bell. These affect the loudness of the bell and the ease with which the ringer can achieve accurate timing during change-ringing. Both these quantities are determined by the first flight of the clapper after it leaves the surface of the bell; they are not influenced by the details of clapper bouncing. It has been shown in earlier work (King et al. [3]) that under reasonable approximations, this free flight of the bell and clapper is governed by just two dimensionless parameters, formed from ratios between three lengths: the equivalent pendulum lengths of the bell and clapper and the offset between the pivot axes of bell and clapper. This will be discussed in some detail in Section 3.1. Plotting the behaviour in the plane of these two parameters leads to useful design charts.

Other relevant issues are influenced by the bouncing of the clapper. These include the frequency bandwidth of the impact, determining how many bell modes are excited, and the envelope of the vibration following the first strike. These bring in two additional dimensionless parameters: the clapper-to-bell mass ratio and the coefficient of restitution on impact. Measurements of the coefficient of restitution have been made on a range of bells, giving values that are consistently very low; less than $10 \%$ of the initial energy is recovered after the first bounce. It will be argued that this low value is caused primarily by the energy used to excite vibration of the bell, and an estimate of the excitation bandwidth will be derived by a simple argument based on energy conservation.

Two less obvious questions about bell dynamics will also be investigated. The first is the phenomenon of "ringing right" versus "ringing wrong." In both diagrams in Figure 1, the clapper is shown resting against the side of the bell that will be the trailing edge during the next swing, so that the clapper catches up with the bell during the swing in order to strike, and it ends up resting on the opposite side. This then becomes the trailing edge for the next stroke. This is "ringing right." But sometimes the clapper will start on the opposite side, the leading edge during the swing, so that the bell catches up with the clapper rather than vice versa during the swing. This is "ringing wrong" and is generally regarded as an undesirable condition since it is harder for the ringer to control the precise moment of striking. Some bells allow both types to occur, depending on the initial setup, while others allow only one or the other. The masses, rotational inertias, and geometric configuration of the bell and clapper make the difference, and it is useful to be able to predict the possible behaviour of a bell from knowledge of these details. A design chart for this purpose, in the parameter plane described earlier, was produced in 1965 [3] based on calculations using a PACE analogue computer of the time. This chart is revisited using digital simulation. This confirms the accuracy of the earlier results and adds significant additional detail.

The second issue to be investigated concerns "double striking" or "double clappering," when a listener is aware of two (or more) strike sounds from the bell on each note. Given that there are always in fact multiple strikes because of clapper bouncing, the key question is why the effect is usually not heard. It will be suggested that the answer relates to a psychoacoustical phenomenon called "echo suppression." A simple perceptual test has been carried out to characterise the conditions under which experienced ringers describe a 
TABLE 1: Measured parameters for the laboratory bell and the (old) tenor bell of Great St Mary's, Cambridge.

\begin{tabular}{lcc}
\hline & Lab & GSM tenor \\
\hline$M(\mathrm{~kg})$ & 45.8 & 1378 \\
$m(\mathrm{~kg})$ & 1.65 & 24.2 \\
$T_{b}(\mathrm{~s})$ & 1.23 & 2.33 \\
$T_{c}(\mathrm{~s})$ & 0.95 & 1.76 \\
$L_{b}(\mathrm{~m})$ & 0.376 & 1.35 \\
$L_{c}(\mathrm{~m})$ & 0.224 & 0.770 \\
$a(\mathrm{~m})$ & 0.24 & 0.705 \\
$b(\mathrm{~m})$ & 0.25 & 0.589 \\
$\left.I_{b}(\mathrm{~kg} \mathrm{~m})^{2}\right)$ & 4.1 & 1311 \\
$\left.I_{c}(\mathrm{~kg} \mathrm{~m})^{2}\right)$ & 0.093 & 11.0 \\
$r(\mathrm{~m})$ & 0.10 & 0.179 \\
$\phi_{\max }(\mathrm{degrees})$ & 26 & 32 \\
$r / L_{c}$ & 0.45 & 0.23 \\
$L_{b} / L_{c}$ & 1.68 & 1.75 \\
\hline
\end{tabular}

sound as a double strike, and this allows predictions to be made of the conditions likely to produce this undesirable phenomenon.

\section{Experimental Results}

2.1. Laboratory Bell. To see what actually happens when a bell is rung, a small bell was mounted in the laboratory on a makeshift "bell tower" so that it could be rung in the conventional way. Some details of this bell are given in Table 1. It is a bronze bell of conventional shape, having a traditional wrought iron clapper with a spherical striking ball. Sensors were used to give information about dynamic interactions; accelerometers were attached to both bell and clapper, and also a simple electrical circuit was designed in which the bell-clapper contact acted as a switch, allowing times of contact and noncontact to be detected directly. Typical results are shown in Figure 2(a). The top trace shows the signal from the bell accelerometer, the middle shows the clapper accelerometer, and the lower trace shows the contact detector; the higher level denotes "out of contact" and the lower level "in contact."

At the left-hand side of Figure 2(a), the clapper is out of contact with the bell. At about $t=0.15 \mathrm{~s}$, the clapper first makes contact, and both accelerometer traces show vibration starting at that moment. The contact trace and the clapper accelerometer then show very clearly a sequence of later impacts, with decreasing magnitude in the acceleration. Inspection of the data on a greatly zoomed scale, not reproduced here, reveals that the duration of the individual contacts is typically in the range $200-500 \mu \mathrm{s}$. By about $t=0.95 \mathrm{~s}$, the clapper has ceased to bounce and is resting in contact with the bell. This continues until about $t=1.3 \mathrm{~s}$, when the clapper lifts out of contact and is in flight ready for the next impact on the next swing of the bell. The "fuzzy" appearance of the contact signal near the moment of liftoff is probably an electrical resistance artefact arising from surface contamination when the contact pressure is very light. Figure 2(b) shows corresponding measurements on the next strike of the bell, in the reverse direction. The pattern is similar but the details are somewhat different; in particular, the time intervals between the first three bounces of the clapper are longer. This will turn out to have a significant implication for the perception of the sound of this bell, as will be discussed in Section 4.3.

The bell acceleration in Figures 2(a) and 2(b) does not show the complicated bouncing activity so clearly. The bell vibrates after the first impact, modified in detail by the later impacts. The bell vibration dies down somewhat during the multiple bouncing, and then just after the time the clapper has come to rest, the remaining vibration in the bell can be seen to die away more rapidly. If the same bell is struck with a single clean impact, as shown in Figure 2(c), the acceleration trace shows a much longer and cleaner decay. This plot was obtained by ringing the bell at low amplitude rather than full-circle.

More detail of the bell vibration is revealed by timefrequency analysis as shown in Figure 3. These plots were generated by short FFTs of overlapping segments of the time data, using a Hanning window on each (see Hodges et al. [4]). The two plots correspond directly to Figures 2 (a) and 2(c). Both show the strongest response at the same frequencies, representing resonant modes of the bell. Analysis of the results in Figure 3(b) allows frequencies and modal $Q$ factors to be extracted; the three strongest components are $624 \mathrm{~Hz}(Q=1300), 981 \mathrm{~Hz}(Q=1000)$, and $1310 \mathrm{~Hz}(Q=2000)$. The single-struck bell shows clear and accurate exponential decay for each of the strongly excited modes, modulated by some evidence of beating. This arises because nearly all "resonances" of an axisymmetric structure like the bell consist of pairs of modes varying with azimuthal angle $\gamma$ according to $\sin n \gamma$ and $\cos n \gamma$ with some integer value $n$. In practice, the two modes are always slightly separated in frequency because of inevitable departures from perfect symmetry, leading to beating.

Figure 3(a) contains the same strong frequencies, but with profiles through time that are more complicated. It also shows more "clutter" in between the resonant frequencies than Figure 3(b). This clutter is presumably caused by the response to multiple impacts from the bouncing clapper, together with some additional input from forces generated at the bearings by the bell and clapper rotation. This bell has an old-style clapper pivot, consisting of a leather strap around a somewhat worn and rusty iron rod. This generates higher and less steady friction forces than a modern bearing.

Figure 4 shows the decay profiles from Figures 3(a) and $3(\mathrm{~b})$ at the resonant frequency $624 \mathrm{~Hz}$. The single-struck bell shows an exponential decay. The circle-rung bell shows an irregular profile which more or less tracks this exponential until about $t=0.8 \mathrm{~s}$, the time when clapper bouncing ceases, after which it shows a very rapid decay. Some comments can be made about possible mechanisms underlying this profile. First, there will be some energy loss associated with each impact; possible mechanisms could include local plastic deformation and air being squeezed out of the contact zone. Second, each impact event will serve to redistribute energy across the frequency spectrum. If energy is transferred from 


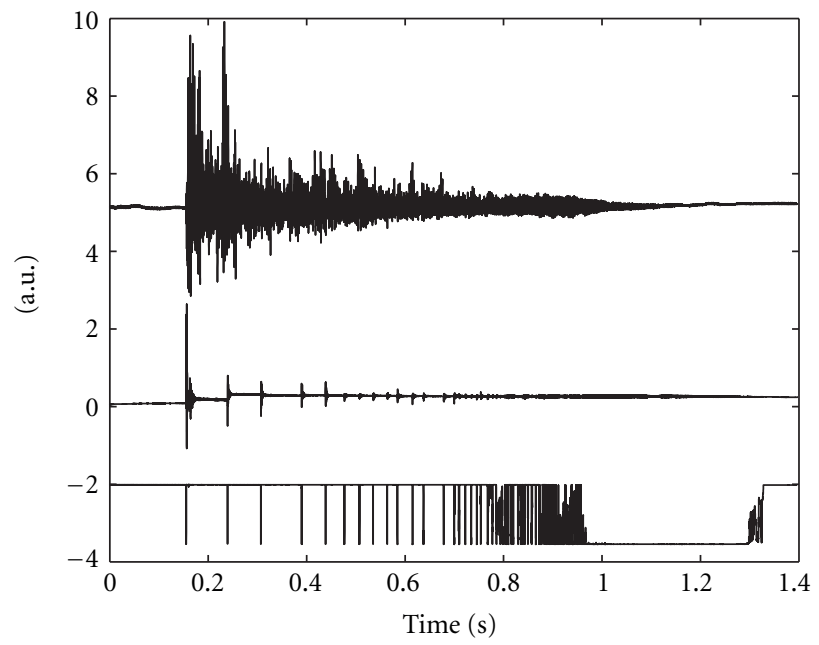

(a)

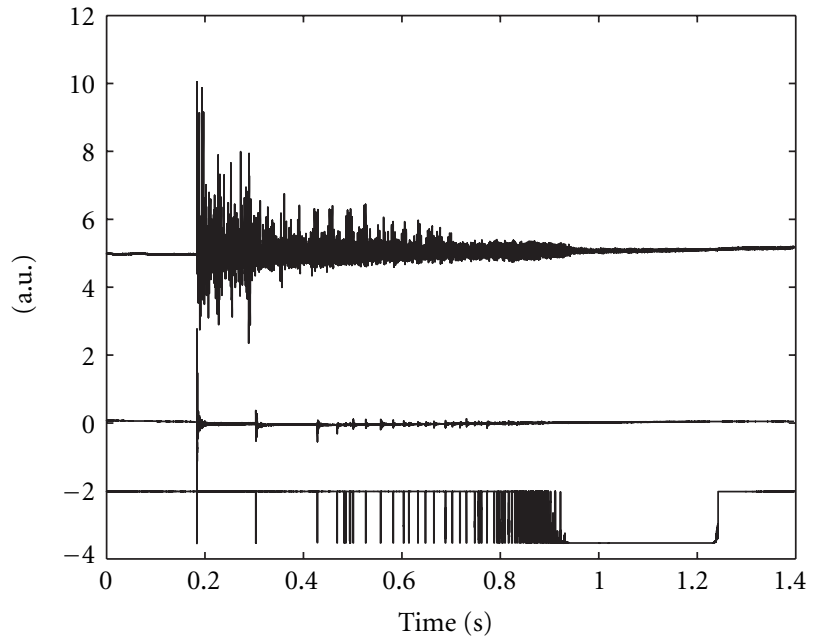

(b)

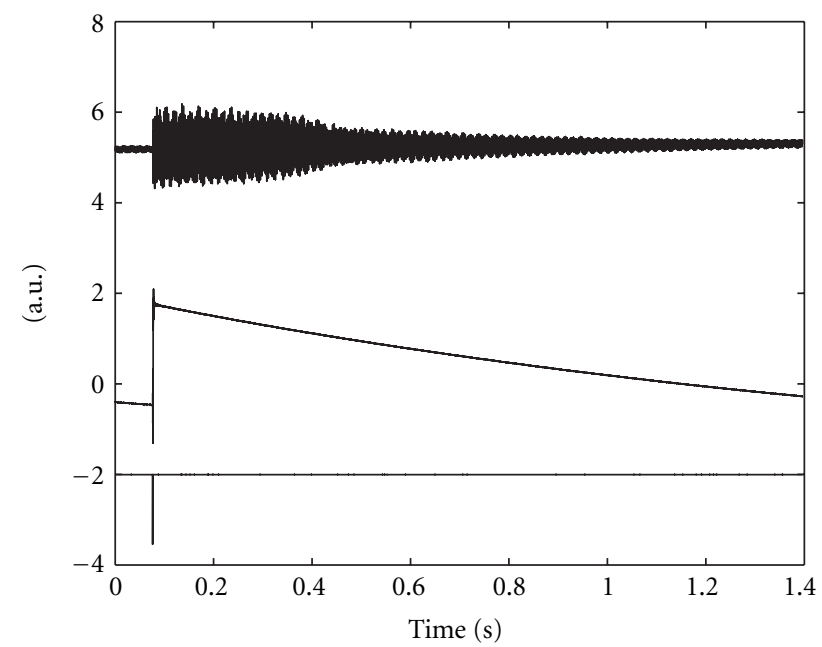

(c)

Figure 2: Measured results on the laboratory bell, showing in each case the outputs of the bell accelerometer (upper trace), clapper accelerometer (middle trace), and contact detector (lower trace). Vertical scale is arbitrary, and traces are vertically separated for clarity. For the contact signal, the lower level denotes "in contact" and the higher level "out of contact." (a) Typical handstroke; (b) typical backstroke; (c) result of a single impact during ringing down.

a slowly decaying low frequency mode to higher modes with faster decay, the overall decay rate will be increased. These effects will influence the somewhat irregular initial period of decay. However, neither of these mechanisms can operate once the clapper has come to rest. The strongest candidate for the observed dissipation after that time is friction. Bending vibration in the rather thick-walled bell will produce some tangential surface motion and thus cause sliding against the resting clapper. It is hard to see at the scale of the plot in Figure 2, but the clapper accelerometer shows significantly raised levels during the period of the rapid decay of the bell, continuing for some $0.3 \mathrm{~s}$.

It seems likely that the relatively long time during which the clapper bounces plays an important, and somewhat counterintuitive, role in the sound of the bell. The sound of a church bell rung full circle is significantly different from the sound of the same bell "chimed" with a clean impact, for example when the bell is used as part of a clock chime. This sound difference is influenced by factors not relevant to this study, especially the Doppler effect of the moving bell, but the behaviour just revealed surely plays a part. If the clapper "stuck" to the bell surface immediately on first impact, without the bouncing, the frictional damping effect would come into play immediately and the sound would be deadened. However, while the clapper is bouncing, the bell sound is able to ring on roughly as it would if chimed; it only switches to the faster decay when the clapper comes to rest. The frictional sliding effect then damps the sound out rather abruptly, in time for the next strike to be heard clearly without much residual vibration from the previous strike.

The suggestion that at the end of each struck note there is a period of frictional "scrubbing" between the clapper and 

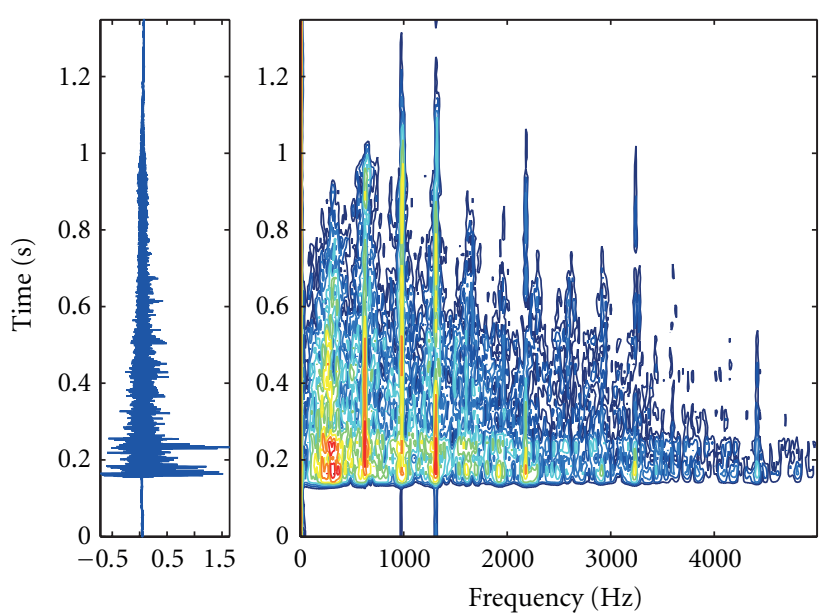

(a)
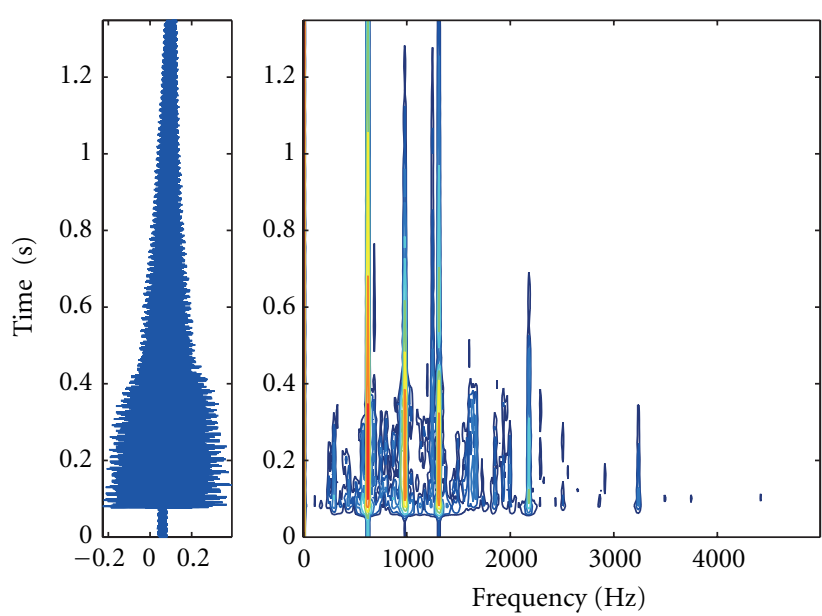

(b)

FIGURE 3: Time-frequency analysis of the bell accelerometer outputs from (a) Figure 2(a) and (b) Figure 2(c). The original time record is reproduced at the left of each plot. Amplitude is plotted using logarithmically spaced contours, with a total dynamic range of $40 \mathrm{~dB}$ down from the highest value.

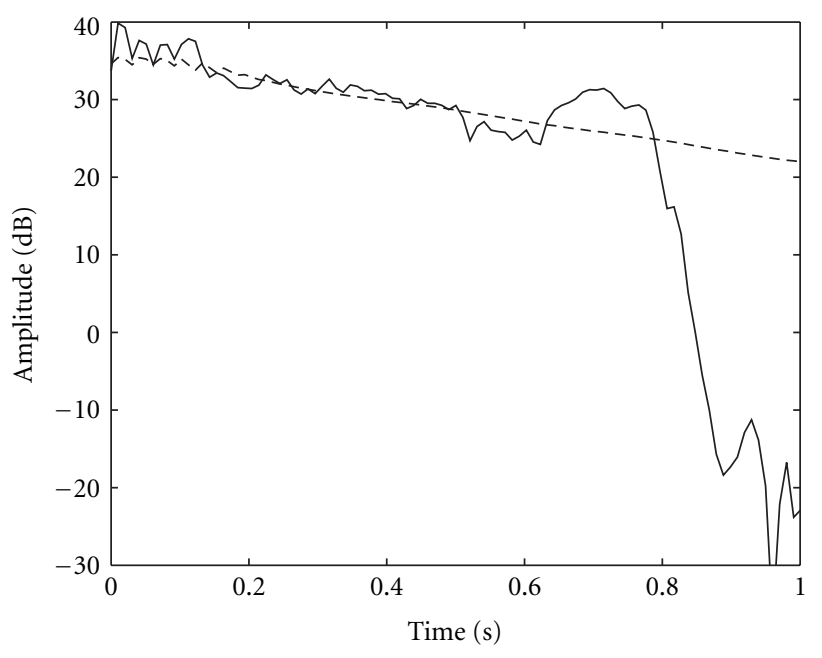

Figure 4: Time decay traces from Figure 3(a) (solid line) and Figure 3(b) (dashed line) for the frequency bin centred on $624 \mathrm{~Hz}$.

the bell may be important for the wear that occurs over time at the striking points on the bell. Bells are made of alloys with very low damping, which generally goes with high hardness (because both are determined by the mobility of dislocations). After an initial "running in" period of a new bell, one would not in fact expect plastic deformation to occur on every strike because work hardening and residual stresses will have been generated at the points of actual contact, just enough to keep them under the elastic limit of the material, a process known as elastic shakedown (see e.g., Johnson [5]). Although at first sight it seems reasonable that the constant hammering of the clapper against the bell would be responsible for the visible wear patch, in fact, frictional wear seems a stronger candidate. Note that matters are quite different for a carillon bell; the frictional effect is then negligible and impact is presumably the main mechanism for generating a wear patch, as has been investigated by Fletcher et al. [6] and Klemenc et al. [7].

2.2. Tests on Other Bells. When the results illustrated in Figure 2 were first obtained, some scepticism was expressed about whether the clapper bouncing was normal behaviour or whether it only happened on atypically small bells like the one tested. To investigate, tests were carried out on two of the bells in Great St Mary's church in Cambridge. From a ring of 12 bells, the tenor (the largest bell) and the fifth bell were selected to give a large and a medium-sized bell to complement the results on the small laboratory bell. It was not possible to install the full range of instrumentation; fitting accelerometers safely on these large bells in a way that allowed full-circle ringing proved impractical. However, the electrical contact measurements could be made simply and safely.

This electrical measurement is sufficient to demonstrate that multiple clapper bouncing occurs during normal ringing with both bells. Example results are shown in Figure 5. The smaller bell in Figure 5(a) shows bouncing for over $1 \mathrm{~s}$ following the initial impact, while the tenor bell in Figure 5(b) shows almost $2 \mathrm{~s}$ of bouncing. The small laboratory bell had about $0.8 \mathrm{~s}$ of bouncing on both handstroke and backstroke, as revealed by Figure 2. It seems that larger bells exhibit the effect more, not less, than the small bell. Bouncing obviously needs to be taken into account in any realistic model of the dynamical behaviour of tower bells.

\section{Theoretical Modelling}

3.1. Governing Equations. The system to be analysed is shown in Figure 6. The bell (plus its supporting hardware) has mass $M$ and moment of inertia $I_{b}$ about its bearing, and the centre of mass lies a distance $a$ from its swing axis. The 


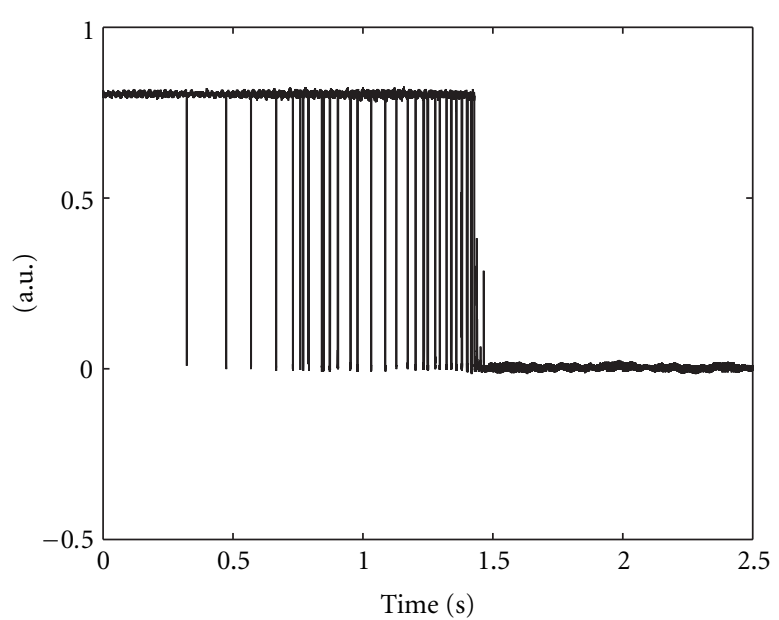

(a)

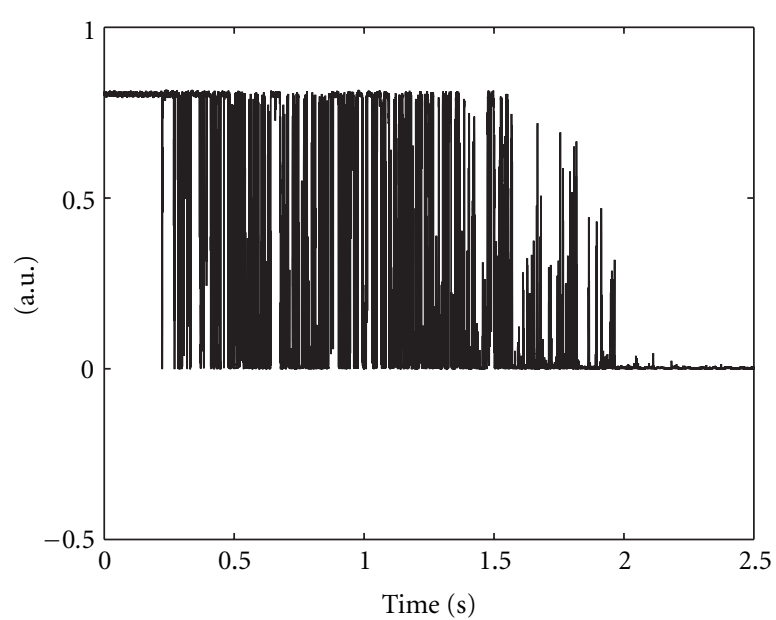

(b)

FIGURE 5: Output from the contact detector for (a) the fifth and (b) the tenor bell from the old ring at Great St Mary's church in Cambridge.

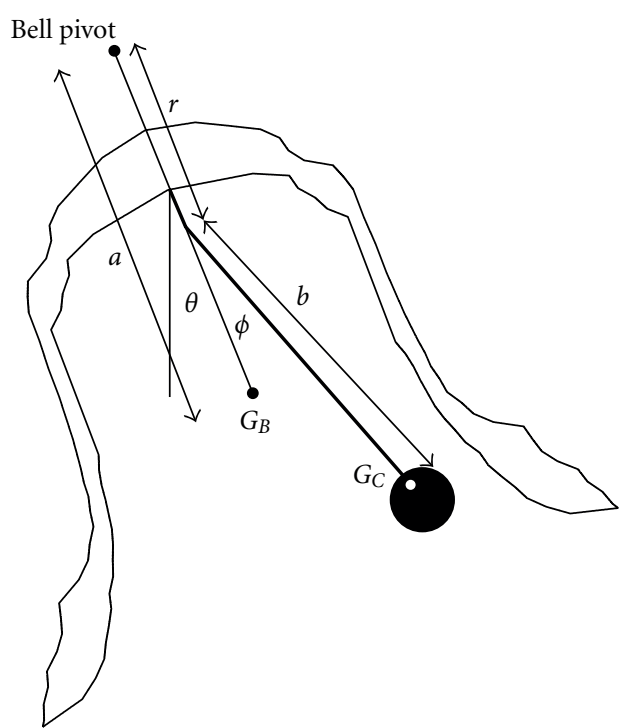

Figure 6: Diagram of a bell and clapper to define notation. The profile is based on measurements of the laboratory bell, but the plot takes a rather coarse form because of the sparseness of these measurements.

clapper has mass $m$ and moment of inertia $I_{c}$ about its pivot, and the centre of mass lies a distance $b$ from its swing axis. The bell and clapper swing axes are separated by a distance $r$, which is shown positive in Figure 6 but is sometimes made negative by hanging a bell in a different configuration. Motion is described by two generalised coordinates: the angle $\theta$ between the bell's axis and the downward vertical and the angle $\phi$ between the clapper and the bell's axis. Effects from the mass of the rope and the ringer's rope force are neglected. Damping in the pivots is also ignored. Finally, the smallamplitude vibration of the bell and clapper is ignored in this modelling of the dynamics of the ringing process, although it will be seen later that the energy involved in vibration enters the problem indirectly through the value of the coefficient of restitution.

The potential energy of the system is

$$
\begin{aligned}
V= & M g a[1-\cos \theta]+m g r[1-\cos \theta] \\
& +m g b[1-\cos (\theta+\phi)],
\end{aligned}
$$

where $g$ is the acceleration due to gravity. Noting that the centre of mass of the clapper has velocity components in Cartesian axes

$$
\mathbf{v}_{g}=\left[\begin{array}{c}
r \dot{\theta} \cos \theta+b(\dot{\theta}+\dot{\phi}) \cos (\theta+\phi) \\
r \dot{\theta} \sin \theta+b(\dot{\theta}+\dot{\phi}) \sin (\theta+\phi)
\end{array}\right],
$$

the kinetic energy is

$$
\begin{aligned}
T= & \frac{1}{2}\left(I_{b}-M a^{2}\right) \dot{\theta}^{2}+\frac{1}{2} M a^{2} \dot{\theta}^{2}+\frac{1}{2}\left(I_{c}-m b^{2}\right)(\dot{\theta}+\dot{\phi})^{2} \\
& +\frac{1}{2} m\left|\mathbf{v}_{g}\right|^{2}
\end{aligned}
$$

which simplifies to

$$
\begin{aligned}
T= & \frac{1}{2} I_{b} \dot{\theta}^{2}+\frac{1}{2} I_{c}\left(\dot{\theta}^{2}+\dot{\phi}^{2}+2 \dot{\theta} \dot{\phi}\right)+\frac{1}{2} m r^{2} \dot{\theta}^{2} \\
& +m r b\left(\dot{\theta}^{2}+\dot{\theta} \dot{\phi}\right) \cos \phi .
\end{aligned}
$$

Applying Lagrange's equation gives the two equations of motion

$$
\begin{aligned}
& {\left[I_{b}+I_{c}+m r^{2}+2 m r b \cos \phi\right] \ddot{\theta}+\left[I_{c}+m r b \cos \phi\right] \ddot{\phi}} \\
& \quad-m r b \dot{\phi}(2 \dot{\theta}+\dot{\phi}) \sin \phi+M g a \sin \theta+m g r \sin \theta \\
& \quad+m g b \sin (\theta+\phi)=Q_{\theta}, \\
& I_{c}(\ddot{\theta}+\ddot{\phi})+m r b \ddot{\theta} \cos \phi+m r b \dot{\theta}^{2} \sin \phi \\
& \quad+m g b \sin (\theta+\phi)=Q_{\phi},
\end{aligned}
$$


where $Q_{\theta}, Q_{\phi}$ are the appropriate generalised forces. During free flight of bell and clapper, these generalised forces are zero, but during contact between clapper and bell, there is a contact force that must be expressed through the generalised forces. This will be discussed in the next subsection.

These equations of free motion can be used in their full complexity for numerical calculations, but in order to understand the dominant aspects of the underlying physics, it is useful to note that they can be simplified if some reasonable approximations are made. The mass and moment of inertia of the bell are invariably much greater than those of the clapper; for the bells studied here, Table 1 shows a mass ratio of 28:1 for the small laboratory bell and 57:1 for the Great St Mary's tenor. Equation (5) consists of an additive combination of terms proportional to these various masses and moments of inertia. If the terms involving $m$ and $I_{c}$ are neglected, this equation takes the far simpler form

$$
I_{b} \ddot{\theta}+M g a \sin \theta \approx Q_{\theta}=0 .
$$

It is now useful to cast (6) and (7) into a nondimensional form. Define

$$
L_{b}=\frac{I_{b}}{M a}, \quad L_{c}=\frac{I_{c}}{m b},
$$

where $L_{b}, L_{c}$ are the lengths of the equivalent simple pendulums with periods of small oscillation matching those of the bell and clapper separately. These periods $T_{b}, T_{c}$ are given by

$$
T_{b}=2 \pi \sqrt{\frac{L_{b}}{g}}, \quad T_{c}=2 \pi \sqrt{\frac{L_{c}}{g}} .
$$

Now, introduce a nondimensional time-like variable based on the bell's period

$$
\tau=t \sqrt{\frac{g}{L_{b}}} .
$$

Combining these substitutions, the equations during free flight of the bell and clapper become

$$
\begin{gathered}
\theta^{\prime \prime}+\sin \theta \approx 0, \\
\theta^{\prime \prime}+\phi^{\prime \prime}+\frac{L_{b}}{L_{c}} \sin (\theta+\phi)+\frac{r}{L_{c}} \theta^{\prime 2} \sin \phi+\frac{r}{L_{c}} \theta^{\prime \prime} \cos \phi=0,
\end{gathered}
$$

where prime denotes differentiation with respect to $\tau$. This form makes it clear that the free motion depends only on two dimensionless parameters: $L_{b} / L_{c}$ and $r / L_{c}$. Both are easy to estimate for bells in situ in a tower; $r$ can be measured directly, and periods of small oscillation can be determined by timing a few cycles, then (9) can be used to obtain $L_{b}, L_{c}$. The behaviour in the plane determined by these two parameters will be discussed in Section 4 .

3.2. Contact Force and Coefficient of Restitution. The modelling just described is uncontroversial and represents the underlying physics of the swinging bell and clapper quite accurately. However, the modelling of the detailed forces and deformations occurring near the contact during an impact is far less simple. Fortunately, it is not necessary to represent these details accurately in order to obtain a satisfactory simulation model for the purposes of this paper. As is clear from the results of Figure 2, the contact time during a single bounce of the clapper is always very short compared to the time scales characterising bell and clapper motion. The contacts last less than $1 \mathrm{~ms}$, and this means that provided the correct transfer of energy and momentum is modelled, each impact could be treated as an instantaneous event.

However, for the purposes of numerical simulation, such an instantaneous event is not convenient. It is far more reliable to use an approach that gives a finite contact force and a continuous variation of the dynamical quantities $\dot{\theta}, \dot{\phi}$ through the impact. To this end, the contact interaction between clapper and bell can be modelled as a simple linear contact spring with a stiffness $k$ defined so that the stored potential energy in the spring is

$$
\frac{1}{2} k\left(\phi-\phi_{\max }\right)^{2} \quad \text { for } \phi>\phi_{\max }
$$

where $\phi_{\max }$ is the maximum value of $\phi$ before contact occurs. There is a corresponding form for negative $\phi<-\phi_{\max }$. During the first part of each contact, the clapper compresses this spring, then it bounces back as the spring extends again. Within the terms of the model developed in the previous subsection, the bouncing behaviour is then very simple; the relative motion of clapper and bell during contact occurs at the "contact resonance" frequency determined by the stiffness $k$ together with the mass of the clapper. As first explained by Rayleigh when talking about the impact of a piano hammer with a string [8], the time of contact is then simply a half-period of this contact resonance oscillation. The reason is that after a half period, the spring force would become tensile $\left(\phi<\phi_{\max }\right)$, so in practice this is the moment when loss of contact occurs.

To model the observed short contact time, a very large value of $k$ is required. However, when $k$ becomes sufficiently big, the actual value ceases to matter from the perspective of the predicted motion of bell and clapper; the overall result converges to what would have been obtained with an instantaneous contact using energy and momentum considerations. The choice of $k$ for simulation purposes can thus be based on computational considerations rather on a need to represent the detailed physics accurately. If the value is too big, it can cause problems of excessive computation time because the time step has to be short enough to resolve the contact resonance oscillation. A convenient value was determined using convergence experiments; the lowest stiffness compatible with reproducing the instantaneous bouncing behaviour was chosen.

The usual virtual work argument for calculating generalised forces can be applied to expression (12), with the result that

$$
Q_{\theta}=0, \quad Q_{\phi}=-k\left(\phi-\phi_{\max }\right), \quad \text { for } \phi>\phi_{\max } .
$$

An interesting consequence of (11) and (13) can be deduced immediately. The measurements in Figures 2(a) and 2(b), 


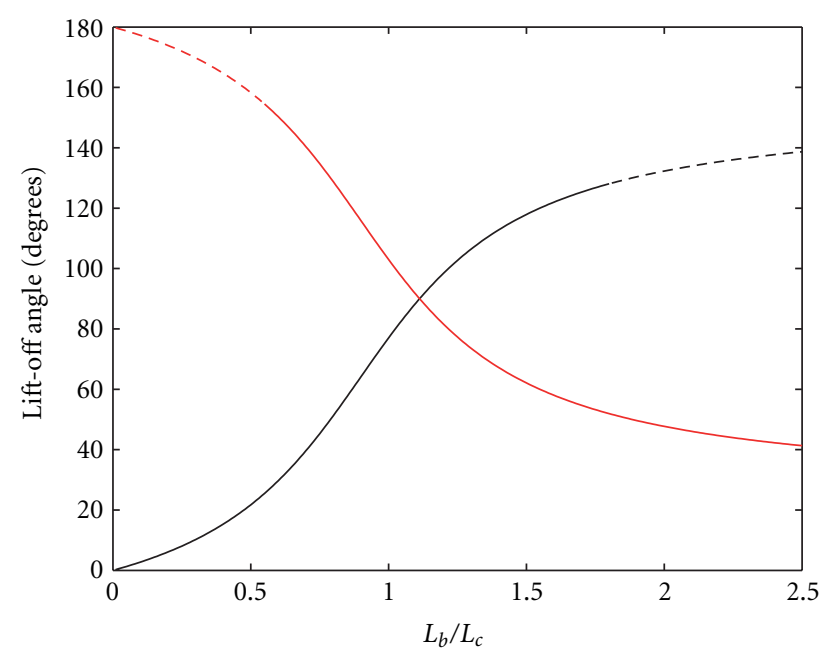

Figure 7: Lift-off angles $\theta_{r}$ (black line) and $\theta_{w}$ (red line) as a function of $L_{b} / L_{c}$ for the special case of bells hung with $r=0$. Dashed portions of both lines indicate ranges where continued ringing in the given style is not possible (see Figure 12).

to be confirmed by simulation results to be shown shortly, reveal that during normal ringing, whether "right" or "wrong," the clapper "sticks to the bell" during the early part of the downswing. At some stage, it lifts off the bell surface into free flight. The angle $\theta$ at which this occurs can be called the lift-off angle, and it shows an interesting pattern of behaviour. This is revealed most clearly for the case $r=0$. For ringing right with $\theta$ initially having a positive value just below $180^{\circ}$, a sticking clapper will have $\phi=\phi_{\max }$, a constant value. In order to satisfy both of (11) including the nonzero generalised force suggested by (13), the value of this generalised force needs to be

$$
Q_{\phi}=\frac{L_{b}}{L_{c}} \sin \left(\theta+\phi_{\max }\right)-\sin \theta
$$

The moment of lift-off is defined by this generalised force changing sign from compressive to tensile, and so with a little rearrangement, the lift-off angle $\theta_{r}$ must satisfy

$$
\tan \theta_{r}=\frac{\sin \phi_{\max }}{L_{c} / L_{b}-\cos \phi_{\max }} .
$$

The corresponding angle $\theta_{w}$ for ringing wrong follows from assuming that $\phi=-\phi_{\max }$, and so

$$
\theta_{w}=180-\theta_{r}
$$

when the angles are expressed in degrees. The two lift-off angles are plotted as a function of $L_{b} / L_{c}$ in Figure 7.

There is another important issue concerning contact modeling. As will be confirmed by results to be shown later, it is important to take some account of the energy lost during impact. This can be done using a simple approach due to Stronge [9]; the values (13) are applied during the compression phase of contact, but during the rebound phase the stiffness is reduced to $R k$, where $R$ is a factor less than unity. This reduction factor directly represents the fraction of stored energy in the contact spring which is lost. During the rebound phase, the generalised forces are given by (13) but with the stiffness replaced by the reduced value. The factor $R$ is the energy-based coefficient of restitution introduced by Stronge to resolve paradoxical predictions of earlier theories [10].

To use this contact model for numerical simulations, a suitable value of $R$ is needed, so a simple experiment was carried out. As in the ringing tests described earlier, the measurement method was first developed on the laboratory bell and then applied to additional tower bells. Four bells were tested: the tenor, fifth and third at Great St Mary's, and the tenor at the Church of Our Lady and the English Martyrs in Cambridge. This set covered the common range of sizes of tower bells and also included clappers of two types: traditional ones made of wrought iron and more modern ones made of SG (spheroidal graphite) cast iron.

The test procedure was simple. An accelerometer was fitted to the clapper using a clamping device, as in the ringing tests described earlier. With the bell stationary in the "up" position, the clapper was lifted a small distance off the surface of the bell and dropped. The accelerometer signal was recorded by a PC-based data logger and numerically integrated to give a velocity signal. Each impact between clapper and bell was visible as a jump in the velocity, and these jumps were measured for the first few impacts. The jumps could be determined reliably, but the absolute velocities before and after impact could not because of difficulties associated with the instrumental hardware and with numerical integration. Absolute calibration of the sensor was not necessary, because the coefficient of restitution can be estimated using only ratios of the velocity jumps, as will now be shown.

Suppose that in the initial impact the clapper falls with velocity $v_{0}$ (defined positive downwards) and rebounds from the stationary bell with velocity $-v_{1}$. In the absence of friction at the clapper pivot, it will arrive back for the second impact with velocity $v_{1}$ and rebound with velocity $-v_{2}$. The first two measured velocity jumps are then

$$
\Delta v_{1}=v_{0}+v_{1}, \quad \Delta v_{2}=v_{1}+v_{2} .
$$

Later velocity jumps can be similarly labelled $\Delta v_{3}, \Delta v_{4}$, and so forth. The factor $R$ defined previously is the ratio of kinetic energies after and before the impact, so that

$$
R=\left(\frac{v_{1}}{v_{0}}\right)^{2}
$$

Then,

$$
\Delta v_{1}=v_{0}(1+\sqrt{R})
$$

Some assumption must now be made about the proportion of energy lost in the second impact. At first sight, it seems natural to assume that this is the same fraction as in the first impact, which would give

$$
\Delta v_{2}=v_{1}(1+\sqrt{R})=v_{0} \sqrt{R}(1+\sqrt{R})
$$




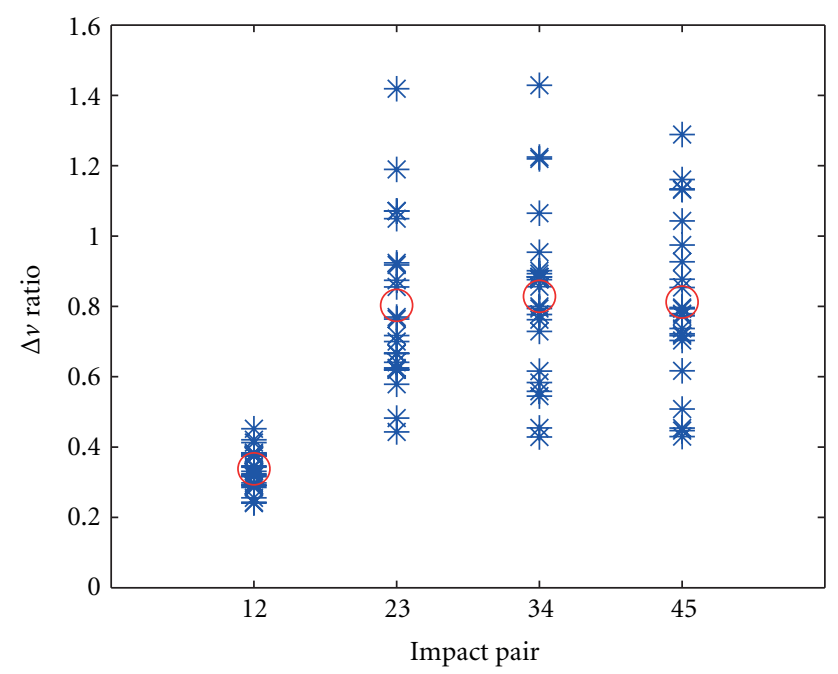

Figure 8: Ratios of velocity jumps at adjacent pairs of impacts, for low-amplitude bounces against a stationary bell as described in the text. The labelling of the horizontal axis indicates the relevant pair of impacts: 1 and 2, 2 and 3, and so on. Data from five different bells are combined. Circles show the mean of each group.

so that $R$ could be estimated from

$$
\frac{\Delta v_{2}}{\Delta v_{1}}=\sqrt{R} \quad \text { or } \quad R=\left(\frac{\Delta v_{2}}{\Delta v_{1}}\right)^{2} .
$$

However, the effective coefficient of restitution that is being used for the bell simulation model is a rather more slippery quantity than this argument acknowledges; the observed pattern of velocity jumps on the real bells is quite different. The results are summarised in Figure 8 for all five bells tested and for a range of drop heights of the clapper on each bell. Every individual velocity jump ratio is shown as a star, and the mean of each set is shown as a circle. The ratio $\Delta v_{2} / \Delta v_{1}$ was always quite small and quite consistent in value, whereas $\Delta v_{3} / \Delta v_{2}$ and subsequent ratios were much more variable and generally significantly bigger, in many cases bigger than unity. It is remarkable how similar the five bells were in this respect. Bell size and clapper material seem to produce no clear effect.

There is a simple interpretation of this pattern. It suggests that a lot of energy is lost on the first impact because it is converted into vibrational energy of the bell-this is, after all, the purpose of the clapper striking the bell. At later impacts, the clapper encounters a bell that is already vibrating with significant velocity normal to the surface at the striking point, in a phase that is effectively random because it is determined by the vibration frequencies, independent of the time between impacts. The bell surface may be approaching or receding from the clapper at the moment of impact, and clapper bounce as observed by the attached accelerometer will be correspondingly enhanced or reduced.

There is obviously no such thing as a single coefficient of restitution that applies to all impacts; the first impact must be treated differently. This suggests an alternative way to interpret the measured velocity jump ratios to estimate $R$ for the first impact. To achieve an estimate at the opposite extreme to (21), it could be assumed that the second impact (and all subsequent ones) is lossless, so that $v_{2}=v_{1}$. Then,

$$
\Delta v_{2}=2 v_{1}=2 v_{0} \sqrt{R}
$$

from which it follows that

$$
\frac{\Delta v_{2}}{\Delta v_{1}}=\frac{2 \sqrt{R}}{1+\sqrt{R}} \quad \text { or } \quad R=\frac{\left(\Delta v_{2} / \Delta v_{1}\right)^{2}}{\left[2-\left(\Delta v_{2} / \Delta v_{1}\right)\right]^{2}}
$$

The mean value of $\Delta v_{2} / \Delta v_{1}$ over all tests on all bells was 0.35 . Using $(21)$ then gives an estimate $R \approx 0.12$, while using (23) gives $R \approx 0.045$. The true answer is somewhere between the two, probably closer to the second estimate, but in any case, it is clear that almost all the energy of the initial clapper motion is absorbed at the first impact, and a very low value of $R$ should be used in simulations.

There is a final twist in this story. The value of $R$ just estimated is only relevant to the first impact. Later impacts will have less energy loss into vibration, but they could only be treated accurately if the bell vibrations were explicitly taken into account so that the true relative velocity of bell and clapper could be determined at each impact. In the simplified analysis based on the equations developed in the previous subsection, vibration is not modelled except through the value of $R$, and in practice, the same value will be used for all impacts. This obviously conflicts with the results of Figure 8, but fortunately the value of $R$ is so low, in the range $0.05-$ 0.1 , that the use of the same $R$ for later impacts will not make much difference to the dynamical interactions, since so little energy is left after the first impact. Simulations for the purpose of sound synthesis might need to consider this question more carefully, but for the present purpose the approximation is thought to be adequate.

3.3. Energy Loss to Vibration at Impact. The measurements just described suggest a rather low value of $R$ of the order of 0.1 or less for the first bounce of the clapper against the bell. This behaviour was tentatively explained in terms of the vibration of the bell (and perhaps the clapper). The bell is more or less quiescent before the first impact, so it seems reasonable that significant energy might be transferred to vibration. As a slight digression from the main thrust of this paper, a simple analysis can be used to assess whether this argument can account for the otherwise unexpectedly low value of $R$. The analysis will turn out to have applications to a wider class of problems than just bell-ringing.

For simplicity, the first impact of the dropped clapper against the stationary bell can be modelled as follows. Treat the clapper head as a mass $m$ with initial velocity $v_{0}$, which rebounds from the bell with velocity $-v_{1}$. The contact force will be a short pulse applied to the bell, with total impulse $I$ satisfying

$$
I=m\left(v_{0}+v_{1}\right)
$$

If that impulse was a perfect delta function, it would evoke the standard impulse response of a linear vibrating system, 
giving a velocity at the contact point that can be written in the form

$$
v(t)=I \sum_{n} u_{n}^{2}\left(x_{0}\right) \cos \omega_{n} t
$$

where $u_{n}\left(x_{0}\right)$ is the magnitude at the impacted point $x_{0}$ of the $n$th mode shape of the bell, with natural frequency $\omega_{n}$. The mode shapes are assumed to be mass normalised, so that

$$
\int u_{n}^{2}(x) d m=1
$$

where $\int \cdots d m$ denotes the appropriate mass-weighted integral over the system, as used in the calculation of the kinetic energy. Note that this normalisation gives $u_{n}$ the dimension

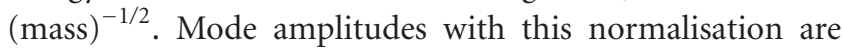
directly measurable; they are the natural result of carrying out experimental modal analysis (Ewins [11]). Damping has been ignored in (25) because the impact duration is so short that any energy dissipation during the contact time will be negligible.

The actual force pulse is not a delta function but has some finite duration. This has the effect of applying a lowpass filter to the mode sum (25). The details are governed by the Fourier transform of the pulse shape, but the result can be visualised approximately as a truncation of the modal sum to neglect natural frequencies higher than the inverse of the pulse duration.

The mode shape factor in (25) is related to the effective mass $M_{n}$ of that mode at that particular point, defined as the mass that, if given the velocity of that point, would have the same total kinetic energy as the vibrating bell. Specifically,

$$
M_{n}=\frac{1}{u_{n}^{2}\left(x_{0}\right)} \text {. }
$$

In terms of these effective masses, each mode excited by the impulse acquires initial velocity $I / M_{n}$ and kinetic energy $I^{2} /\left(2 M_{n}\right)$. Now, an upper limit on the total energy "absorbed" into vibration of the bell can be found by supposing that there is no actual dissipation during the impact, and thus applying conservation of kinetic energy through the impact

$$
\frac{1}{2} m v_{0}^{2}=\frac{1}{2} m v_{1}^{2}+\sum_{n} \frac{I^{2}}{2 M_{n}} .
$$

Substituting I from (24) and defining

$$
\lambda=\frac{v_{1}}{v_{0}}
$$

gives the quadratic equation

$$
1=\lambda^{2}+(1+\lambda)^{2} \sum_{n} \frac{m}{M_{n}}
$$

which has a trivial solution $\lambda=-1$, leaving the solution of interest as

$$
\lambda=\frac{1-K}{1+K}
$$

where

$$
K=\sum_{n} \frac{m}{M_{n}}
$$

If the impulse was a delta function, the infinite sum (32) would give $K \rightarrow \infty$, because the masses $M_{n}$ are finite for almost all $n$; the typical value is of the same order as the actual total mass of the system. But it is clear from this analysis that $K$ cannot in fact be significantly greater than unity if the clapper mass is really to rebound. For a clean rebound, $\lambda$ needs to be positive, or at least can only be very slightly negative. If the clapper is still moving forward after the impact faster than the mean speed imparted to the bell, it will meet the bell surface again almost immediately, and these multiple contacts should probably be combined and regarded as a single event. The case $K=1$ also corresponds to the maximum energy that can be put into vibration of the bell, because all the kinetic energy in the initial clapper motion is transferred to bell vibration. This limit on $K$ imposes a limit on the truncation of the sum (32), in other words it sets a minimum length for the pulse duration. The effective masses $M_{n}$ can be calculated or measured, for a bell or any other structure, using standard methods. Within this simple approximation, the pulse length must be such as to truncate the series before the mode that would take $\sum 1 / M_{n}$ above the value $1 / \mathrm{m}$.

More accurately, this argument could be applied with an assumed pulse shape, such as a half-cycle sine wave as used by Rayleigh [8]. Following through the same analysis, incorporating the appropriate low-pass filter derived from the pulse shape, and evaluating the expression corresponding to (32) as a function of the width of the pulse would lead to a minimum possible value for the pulse width of a clean rebound. Of course, the pulse can always be made longer than this minimum value, for example, by putting a soft spring at the contact point as with the felt facing of piano hammers, but it cannot be made shorter than this value however carefully the surface hardness and the contact geometry might be refined, provided a clean bounce is to occur.

Beyond the application to bell-ringing, this result is relevant to the issue of choosing an impulse hammer to measure vibration response of a structure: to excite modes a long way up the modal series, a hammer must be chosen that is very light compared to the mass of the structure, since increasing the hammer mass acts as a low-pass filter. Another familiar feature is that when pushing to the limit in this way with a light hammer and a hard tip, the experimenter's tapping technique has to be good because the hammer has very little tendency to bounce off of its own accord; all the energy has gone into vibration of the tested structure. Indeed, impacts using miniature hammers with hard tips are very prone to multiple contacts; perhaps the reason is that they have $K>1$, making multiple contacts almost inevitable. A cure would be to use a slightly heavier hammer.

It is straightforward to apply the prediction of this simple argument to the behaviour of the laboratory bell discussed in Section 2.1. The bouncing-clapper tests suggested an initial energy reduction ratio $R$ of the order of 0.1 or less. 
That corresponds to a velocity ratio $\lambda$ of the order of $\sqrt{0.1}=-0.32$, and so from (31), the expected value of $K$ is approximately 0.5 or (probably) greater. The clapper mass is about $1 / 30$ the total mass of the bell. The effective modal mass of a mode driven near an antinode would be expected to be roughly around $1 / 3$ the actual total mass, giving a ratio $m / M_{n}$ of the order of 0.1 . So, we might expect not many more than about 5 modes of the bell to be strongly excited, and certainly no more than about 10 . The time-frequency plot in Figure 3(a) gives quite good confirmation of this estimate. It shows three strongly excited modes, plus a few less strong responses at higher frequencies. Bearing in mind that the rigid-body swinging motion, with a frequency close to zero compared to the acoustic modes, must be counted among the strongly excited modes, an aggregate number of the order of 5 is indeed obtained: surprisingly close agreement given the crude nature of the approximations used.

\section{Simulations}

4.1. Preliminary Results. Equations (5), (6), and (13) determine the motion of the system. They are too complicated to expect analytical solutions even without impact events, but they can be solved straightforwardly by standard numerical procedures. In this study, the Matlab routine ODE45 was used; this proved satisfactory for all the test cases tried, once suitable values were established for the tolerance parameters required by ODE45 to specify the precision of solution. The coupled equations (5) and (6) first need to be manipulated to separate $\ddot{\theta}, \ddot{\phi}$. Although the equations are nonlinear, these two quantities occur only in linear combinations in the two equations, so at each time step a pair of simultaneous equations can be solved using the current values of all other variables. The two second-order equations are then readily separated into four first-order ones, the form required by ODE45.

For each run, the bell is initialised in a stationary position close to the vertical, but far enough from it that it immediately starts to fall; the angle $\theta$ was initialised at $171^{\circ}$ for all simulations unless stated otherwise. The exact value of this initial angle turns out not to be critical for the main conclusions of this study: some remarks on this will be made in Section 4.4 when the initial angle will be deliberately varied. The clapper is initially at rest against one side or the other of the bell; both cases merit study to investigate whether sustained "ringing right" or sustained "ringing wrong" is possible. The simulation then runs for a fixed period of time. Whenever contact between clapper and bell is detected, the contact spring comes into play via the generalised force $Q_{\phi}$. Recall that $Q_{\theta}=0$ always. The chosen value of contact stiffness $k$ was $1 \mathrm{MN} / \mathrm{rad}$, and the reduced stiffness $R k$ was used during the rebound phase to represent energy loss at impact as explained earlier. The first set of simulations to be shown use the value $R=0.2$; other values of $R$ are used later on to explore the effect of the coefficient of restitution.

Some typical results of the simulations are shown in Figure 9. Figure 9(a) shows the behaviour using parameter values appropriate to the small laboratory bell, initialised in the "ringing right" state. This plot then shows the typical signature of ringing right; $\theta$ and $\phi$ generally have the same sign throughout the motion. The plot shows the bell angle $\theta$ varying most of the way around the circle for one handstroke and the following backstroke, from near-vertical back to near-vertical in each case. The clapper is limited between the angles $\pm 26^{\circ}$ for this particular bell. Rather careful examination is needed to see the clapper bouncing in the waveform of clapper relative angle $\phi(t)$ in this plot, but it is much more obvious in the waveform of $\dot{\phi}(t)$, shown as a solid line (with an amplitude scale factor convenient for fitting it to these axes, the same factor being used for all four cases in Figure 9). Each bounce shows as a sudden jump in $\dot{\phi}$, upwards for the handstroke and downwards for the backstroke. The rapidly decreasing series of impacts following the first bounce can be seen clearly.

Figure 9(b) shows the same bell ringing wrong. The waveforms all look similar to Figure 9(a), except that now $\theta$ and $\phi$ generally have opposite signs. The remaining cases shown in Figure 9 relate to bells with different parameter combinations, the details of which will become clear in the next subsection when the "clappering chart" will be presented. Figure 9(c) shows a bell ringing right, with rather high values of $\dot{\phi}$. All other things being equal, this bell will ring louder than the bell shown in Figure 9(a) because its dynamical properties lead to a harder strike of the clapper against the bell. Figure 9(d) shows a bell, again ringing right, in which the first strike occurs very late in the bell's swing; the clapper only just manages to catch up with the bell and hit it as the bell is approaching the vertical. This might represent a bell that would be hard for the ringer to control, because it might fail to strike during a swing with reduced angle as is frequently necessary during change ringing. More light will be shed on this possibility by the charts to be shown in the next section.

4.2. Clappering Charts. As was explained in Section 3.1, to a good approximation the free motion of bell and clapper should be governed by just two dimensionless parameters, $L_{b} / L_{c}$ and $r / L_{c}$. This prediction was tested with the simulation program and found to hold up very well over the range of parameters covering normal bells. This makes it natural to map behaviour of interest into the plane determined by these two parameters, using whatever approach is computationally convenient to achieve parametric variation in that plane. Note that all simulations were carried out using the full equations, in terms of time $t$. The approximate equations and the non-dimensional time $\tau$ are not used. The specific procedure used to scan the parameter plane was as follows. The parameter values $M, m, a$, and $I_{b}$ were all taken from the laboratory bell and clapper as listed in Table 1, and were kept fixed throughout. The period $T_{b}$ was held fixed at $1.8 \mathrm{~s}$ : as seen in Table 1 this period is intermediate between the small laboratory bell and the large Great St Mary's tenor. The corresponding value of $L_{b}$ is $0.85 \mathrm{~m}$. To achieve each particular point in the parameter plane, the length $L_{c}$ was first chosen to achieve the desired value of $L_{b} / L_{c}$, then the 


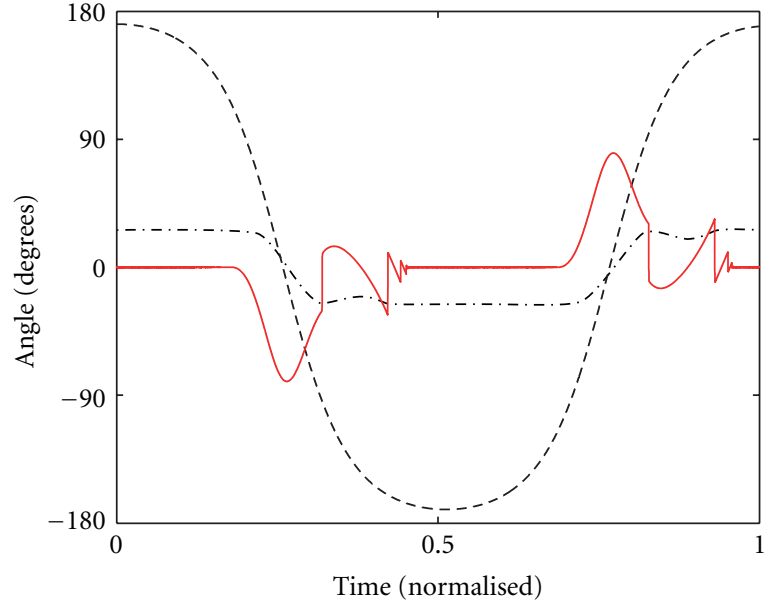

(a)

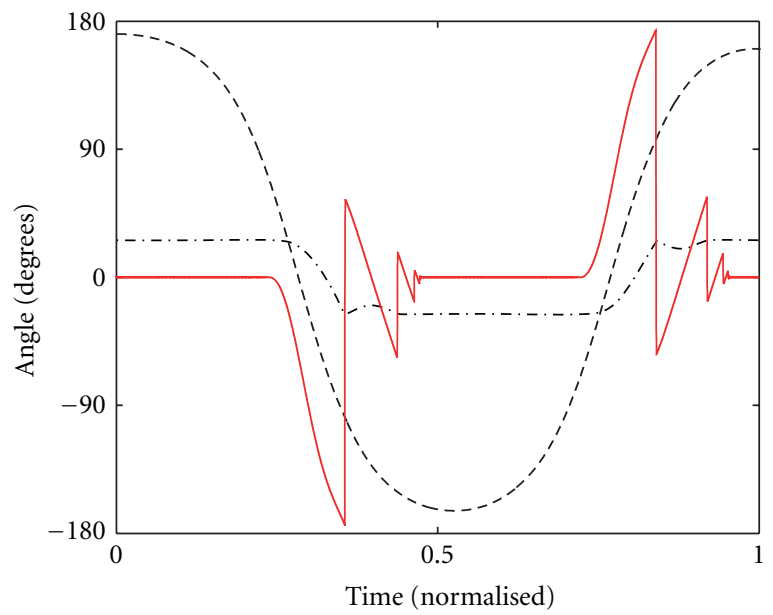

(c)

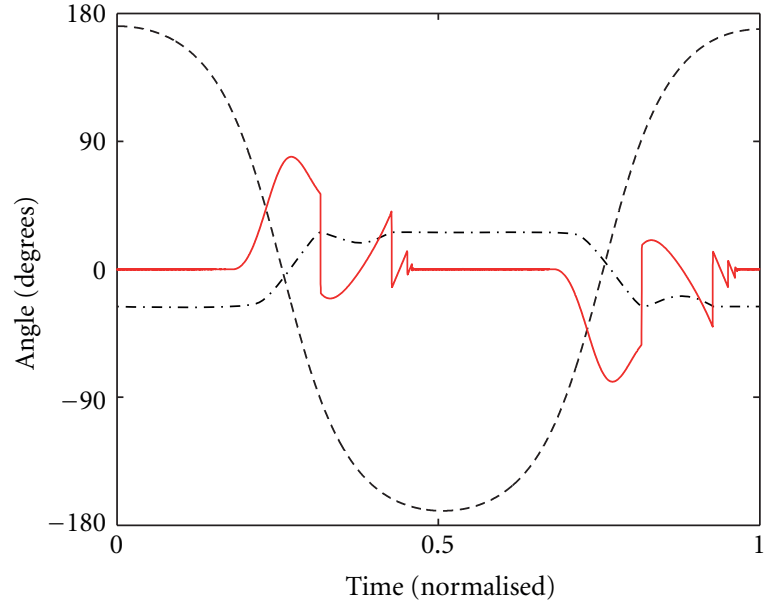

(b)

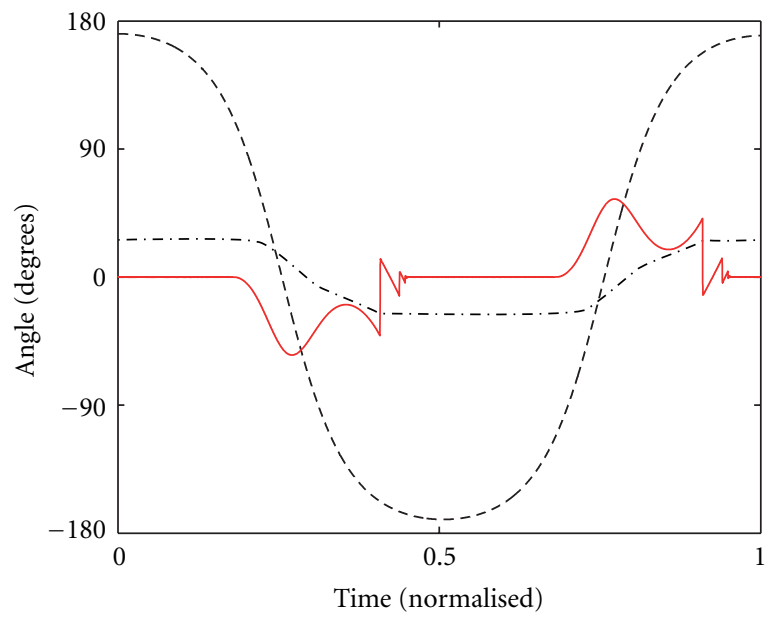

(d)

Figure 9: Typical simulation results, with time scales normalised to the period of a single swing from near-vertical to near-vertical. Each diagram shows bell angle $\theta(t)$ (dashed line), clapper relative angle $\phi(t)$ (dash-dot line), and clapper angular velocity $\dot{\phi}(t)$ (solid red line). The vertical scale applies to the two angles; the angular velocity trace is scaled by a convenient factor to fit to the scales (the same factor in each case). Cases are indexed by the pair of values of $r / L_{c}$ and $L_{b} / L_{c}$ as follows: (a) laboratory bell $(0.446,1.68)$, ringing right; (b) same bell as (a), ringing wrong; (c) bell at $(0.458,0.732)$, ringing right; $(d)$ bell at $(-0.005,1.363)$, ringing right.

offset distance $r$ was chosen to match the value of $r / L_{c}$. The parameter $I_{c}$ was held fixed at the value $0.20 \mathrm{~kg} \mathrm{~m}^{2}$ and the required value of $b$ computed from (8).

In real applications to bell-hanging, the bell period could be changed by, for example, changing the headstock of the bell to "tuck it up". The clapper period could be changed by counterbalancing or otherwise changing the mass distribution. Because the two non-dimensional parameters can be changed by practical interventions of this kind, it is convenient to call the resulting plots "clappering charts." For the purposes of simulation, the limiting value $\phi_{\max }=26^{\circ}$ has been used, measured from the laboratory bell. This angle varies slightly among different bells, but the behaviour is not found to be very sensitive to the exact value, so it is felt that the charts computed here will give a reasonable representation of the behaviour of all normal church bells.
Ranges of the two dimensionless parameters were chosen to match those used in the earlier study [3], and a $20 \times 20$ grid was used to cover this rectangular region of the plane. For each point in this grid, the simulation program was run with initial conditions corresponding to ringing right and ringing wrong. The resulting computed motion was analysed to detect whether the bell was able to continue ringing right, wrong, neither, or both. Impacts were also detected, allowing a number of useful quantities to be computed; relevant to the following discussion are the bell angle $\theta$ at first impact, the clapper relative angular velocity $\dot{\phi}$ just before each impact, and the time delay between the first and second impacts.

Figure 10 shows the value of $\theta$ at first impact for all configurations capable of ringing right (Figure $10(\mathrm{a})$ ) and all capable of ringing wrong (Figure $10(\mathrm{~b})$ ); since $\theta$ was initially positive, first impact normally occurs at a negative angle. Values of zero, and the darkest blue shade, correspond to 


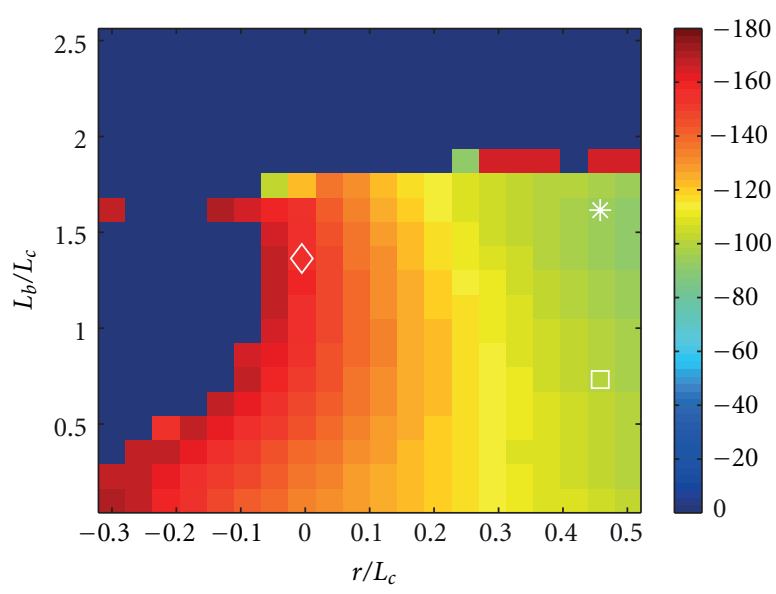

(a)

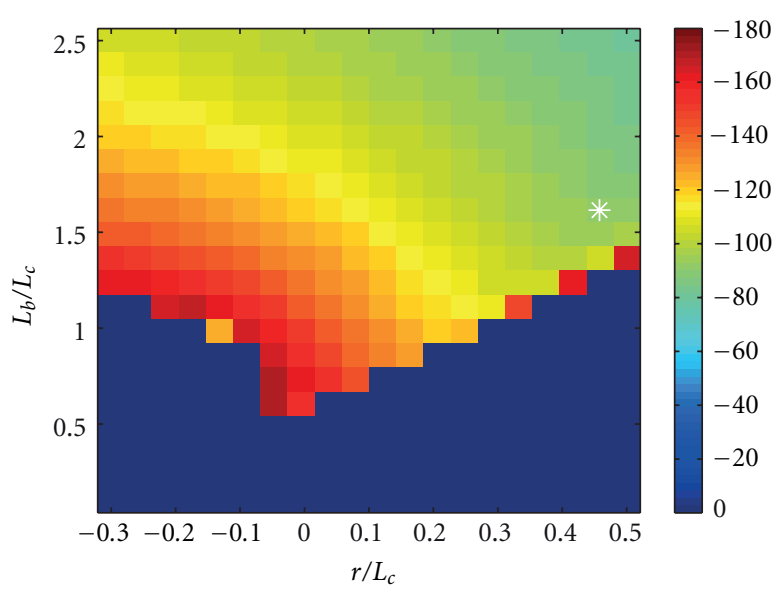

(b)

FIGURE 10: Regions in the "clappering plane" for which a bell can ring (a) right and (b) wrong. Colour shading indicates the bell angle $\theta$, in degrees, at which the first contact occurs between clapper and bell. The bell motion was initialised at angle $\theta=171^{\circ}$. White symbols indicate the positions of the bells from Figure 9, three cases ringing right and one ringing wrong (see text).

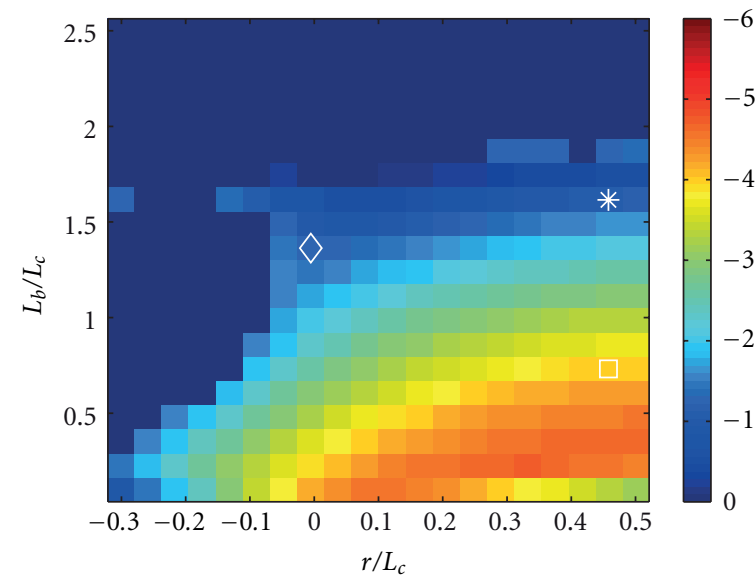

(a)

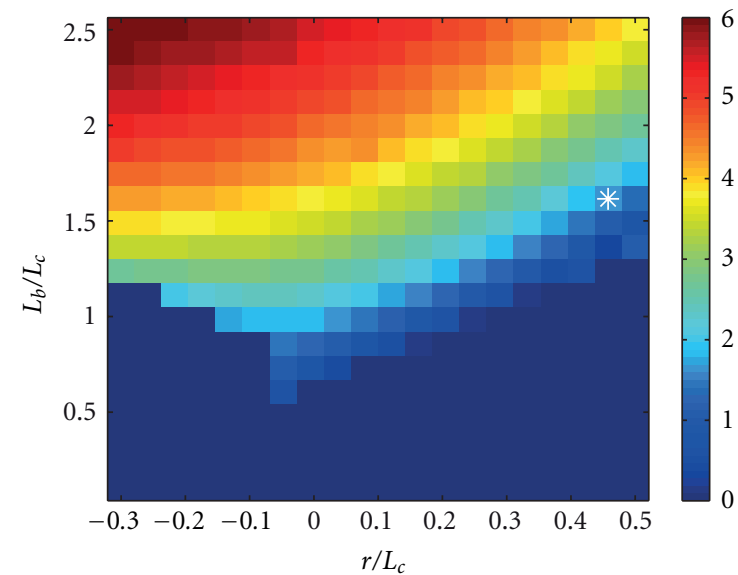

(b)

FIGURE 11: Regions in the "clappering plane" for which a bell can ring (a) right and (b) wrong. Colour shading indicates the clapper relative angular velocity $\dot{\phi}$ at the moment of first contact between clapper and bell. The bell motion was initialised at angle $\theta=171^{\circ}$. White symbols indicate the positions of the bells from Figure 9.

points where the relevant regular ringing was not possible. The boundaries of the "right" and "wrong" regions revealed by this plot have been compared to the graph resulting from the earlier study [3] and found to agree remarkably accurately. This is a tribute to the earlier study, using such a different approach and relatively primitive equipment, and it is also a valuable check on the accuracy of the current simulation model.

Figure 11 shows, in the same format as Figure 10, the value of $\dot{\phi}$ at first impact, which must inevitably be negative for ringing right and positive for ringing wrong. The magnitude of $\dot{\phi}$ translates roughly into loudness of the strike (for a given bell). It is important to note that the numerical value of $\dot{\phi}$ is not the same for different bells occupying the same position on the clappering chart, because the justification for the chart axes was based on the nondimensional time variable introduced in (10). However, the time-scale implicit in $\dot{\phi}$ does not in fact vary over this version of the diagram since it was computed with a fixed bell period $T_{b}=1.8 \mathrm{~s}$, so it should give results representative of typical church bells. Note from Figure 11 that when a given bell is capable of being rung both right and wrong, there is usually a different value of $\dot{\phi}$ at first impact for the two cases. This means that a switch from ringing right to ringing wrong will usually change the loudness of that bell, sometimes drastically, and the ringer has no way to compensate for this.

Figure 11 shows what happens on one set of boundaries in the charts: the upper limit on ringing right and the lower limit on ringing wrong. On both these boundaries, the value of $\dot{\phi}$ at first impact goes to zero. This means that the clapper does not strike the bell and bounce off but makes a "soft landing" on the bell that is in fact the mirror image of the liftoff at the start of clapper flight. There is no transfer of energy, and no strike will be audible. The strike gets progressively 


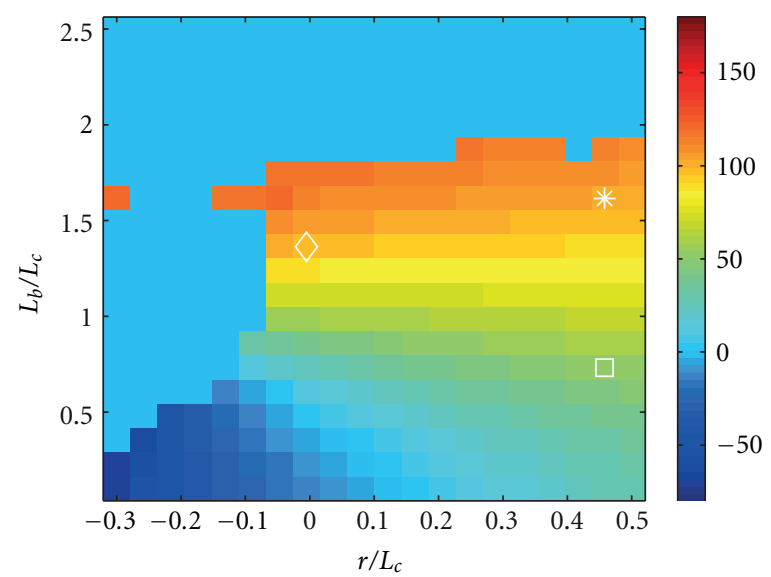

(a)

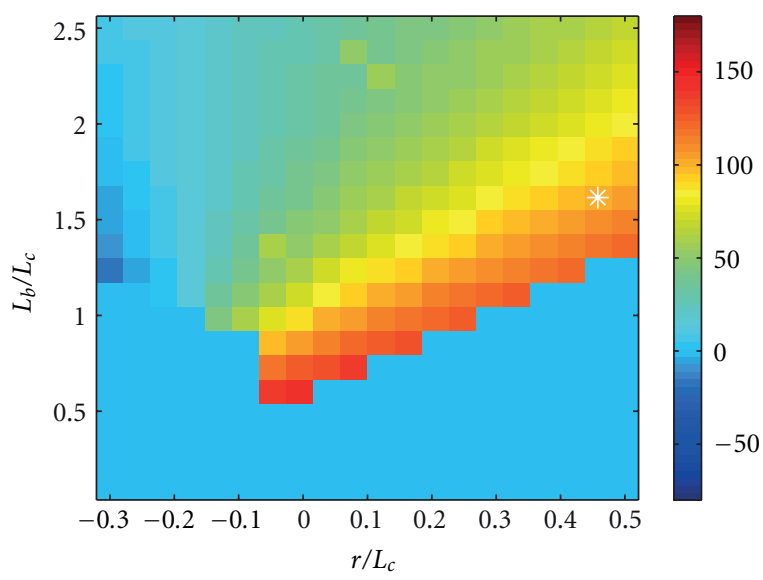

(b)

Figure 12: Regions in the "clappering plane" for which a bell can ring (a) right and (b) wrong. Colour shading indicates the lift-off angle $\theta$ (in degrees) at which the clapper first leaves the surface of the bell on each stroke. White symbols indicate the positions of the bells from Figure 9.

stronger, in either case, as the position in the chart moves further from this boundary into the region where the chosen ringing pattern (right or wrong) can be sustained. Study of the detailed simulation results reveals what happens to the bell and clapper on the other side of these boundaries. The value of $\dot{\phi}$ at first impact increases again, but now after the first impact, the clapper crosses the bell and impacts on the other side during the same swing of the bell. Thereafter, the ringing pattern switches; for example, in Figure 11(a) at a point just above the boundary, a bell that was initially ringing right switches to ringing wrong.

The vertical axis in the clappering charts shows $L_{b} / L_{c}$ and hence determines the period of the bell swing relative to the clapper swing. For ringing right, one might have thought that the clapper needs a shorter period than the bell so that it can catch up, while the converse would be the case for ringing wrong. The plots show the opposite pattern. The reason relates to the fact, noted earlier, that the clapper "sticks to the bell" initially during the down-swing. It does not start its flight until it reaches the lift-off angle, plotted in Figure 7 for the particular case of $r=0$. Towards the bottom of the charts, a bell ringing right shows late lift-off, while the same bell ringing wrong shows very early lift-off. The pattern is reversed at the top of the charts. What is seen in the simulations is the behaviour described earlier: except in pathological cases, if the clapper lifts off very early during the first down-swing, it will strike the opposite side sufficiently early to take up the behaviour it would have had if it had started on that side. It will cross over and strike a second time. If the bell had been set off in a configuration for ringing right, it switches to ringing wrong, and vice versa.

So, early lift-off is a sign of a style of ringing (right or wrong) that is not stable and spontaneously reverses. The pattern of lift-off angles shown in Figure 7 is then consistent with the fact that ringing right tends to occur in the lower part of the clappering chart and ringing wrong in the upper part. The simulation results for the full pattern of liftoff angles for bells ringing right and wrong are shown in Figure 12. The columns in these plots corresponding to $r=0$ confirm the values shown in Figure 7.

The physical condition determining the other main boundaries in the clappering charts, the curving lines on the left-hand side, is revealed by Figure 10. On this boundary, the angle $\theta$ at first impact tends towards the value at the top of the bell's swing, close to $-180^{\circ}$, so that the clapper only just makes contact as the bell reaches the top of its swing. An example close to this boundary was shown in Figure 9(d), indicated by a diamond symbol in Figure 10(a). The laboratory bell is indicated in Figures 10(a) and 10(b) by a star; the model agrees with practical experience that this bell is able to ring either right or wrong. Finally, the square symbol in Figure 10(a) shows the position of the bell illustrated in Figure 9(c); this has roughly the same value of $\theta$ at first impact as the laboratory bell, but Figure 11(a) shows that it has much higher impact velocity.

4.3. Double Striking. The aspects of behaviour discussed so far do not depend on what happens after the first impact, so the value of $R$ used in the simulations makes virtually no difference to the plots. The next aspect to be discussed, however, does depend on the bouncing of the clapper. This is the question of double striking or double clappering. Some bells produce a clear audible impression of a double strike on each stroke. The first question to ask is why this is not the case for all bells, since the results of Figures 2 and 5 show that there are always multiple impacts between the clapper and the bell during normal ringing.

The answer to this probably lies in a psychoacoustical phenomenon known in different manifestations by various names, including "echo suppression" and "forward masking" (see e.g., Moore [12]). The human hearing system has evolved to cope with sounds in the presence of echoes from 


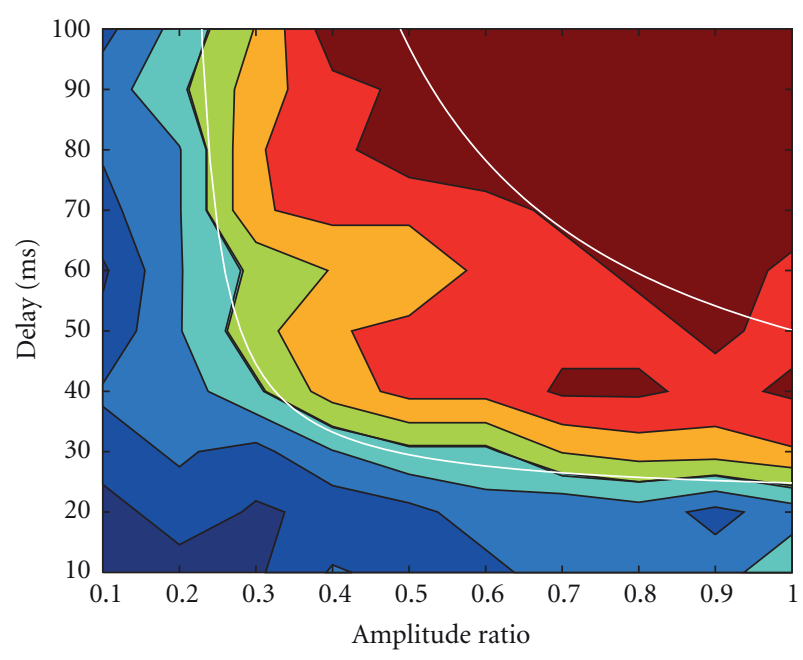

Figure 13: Results of listening test for double striking as described in the text. Average score across all participants is shown with equally spaced contours between +1 (darkest blue) and -1 (darkest red). The heavy black line shows the zero contour and the lower white line the hyperbolic fit to this contour. This corresponds to a value $D=1$ in (33) and represents the threshold of perception. The upper white line corresponds to the value $D=10$, and in the region above this line, almost all subjects hear a double strike.

environmental features like trees or walls. It was presumably important for our distant ancestors to be able to distinguish and locate the actual source of a sound, without being too confused by echoes from other directions. The result is that if we hear a sound followed quickly by a recognisable copy of the same sound, especially if it comes from a different direction, our brains identify the second sound as probably being an echo. We are then, ordinarily, not consciously aware of the echo as a separate event, although it contributes to our sense of the acoustical environment we are in.

The sound of a church bell excited by multiple clapper strikes may tap into this mechanism, so that the later impacts are perceptually discounted to a greater or lesser extent. In the case of the bell, there is no directional difference between the sounds, so the echo suppression effect is less strong than in the case of, for example, wall reflections in an enclosed space. Nevertheless, it seems to be empirically the case that most bells are not perceived as producing multiple strikes. To establish a criterion for the perception of double striking so that it can be explored in a clappering chart requires experimental input.

A simple listening test was conducted in which 19 experienced ringers were played 100 computer-synthesised sounds and, for each one, were asked to say whether they would describe it as a single strike or a double strike. Each sound represented a bell being struck twice; the first strike was the same in each of the 100 tests, while the second was quieter (or at least no louder) because of being scaled down by an amplitude ratio that was varied between the different sounds. The synthesised strike sound was based on the strong frequencies of the laboratory bell. The time delay between the two strikes was also varied between the different sounds. On the basis of preliminary tests, suitable ranges were chosen for both parameters: ten values of amplitude ratio from 0.1 to 1 and ten values of time delay from $10 \mathrm{~ms}$ to $100 \mathrm{~ms}$. A $10 \times 10$ grid of equally spaced values covering these two ranges was used. Each listener was presented with these 100 sounds, in a random order that was different for each test. Sounds were presented via Sennheiser HD580 headphones.

Responses were collected using a Matlab program and processed by a very simple procedure. Each response was allocated the value -1 for "double strike" and +1 for "single strike." The matrix of responses over the test grid was averaged across all subjects, after excluding two outliers. It turned out that the two youngest subjects (both teenagers) produced a very different pattern of responses from the rest. This may point towards an interesting psychoacoustical phenomenon possibly worthy of further study, but for the present purpose, it was judged to be a distraction from the main task. The resulting averaged score in the delay-scale plane is shown as a contour map in Figure 13; contours are equally spaced between +1 (darkest blue) and -1 (darkest red). The zero contour, representing an estimate of the mean threshold of perception for double striking, is marked by the heavy black line: any sound represented by a point below and to the left of this line was perceived by the majority of listeners as a single strike, whereas one above and to the right of the line was perceived by the majority as a double strike.

Based on the results of Figure 13, a rough and ready index of "double strike propensity" can be defined by

$$
D=\frac{(A-0.2)\left(\Delta t_{12}-22\right)}{2.25},
$$

where $A$ is the amplitude ratio of the second strike to the first, and $\Delta t_{12}$ is the time delay in milliseconds between the first and second strikes. This gives contours of equal $D$ that are hyperbolae with asymptotes at $22 \mathrm{~ms}$ delay and 0.2 amplitude ratio, having values greater than unity in the region of perceived double striking in Figure 13. The $D=1$ line is shown in Figure 13 as the lower white curve, matching the threshold line quite well. A second white curve shows the line $D=10$, which very roughly tracks the boundary beyond which everyone agreed that all sounds were double strikes.

This quantity $D$ is easily computed for each simulation case. Results can be plotted as a clappering chart, but a few words of explanation are first needed. Both $A$ and $\Delta t_{12}$ depend on the simulation results beyond the first impact, but the justification for the clappering charts was based on the equations for free flight before any impact. Certainly, the motion after the first impact is not determined purely by the two non-dimensional parameters used in the charts; two further parameters enter, the limit angle $\phi_{\max }$ and the coefficient of restitution $R$. However, for fixed values of these two new parameters, it can be argued that, to a good approximation, the motion is then fully defined by position in the clappering chart. The free-flight equations give the time and relative angular velocity of the first impact. The rebound angular velocity is determined by the value of $R$, then the clapper is in free flight again, so the original equations determine the time until the second impact and the angular velocity with which that occurs. That gives 


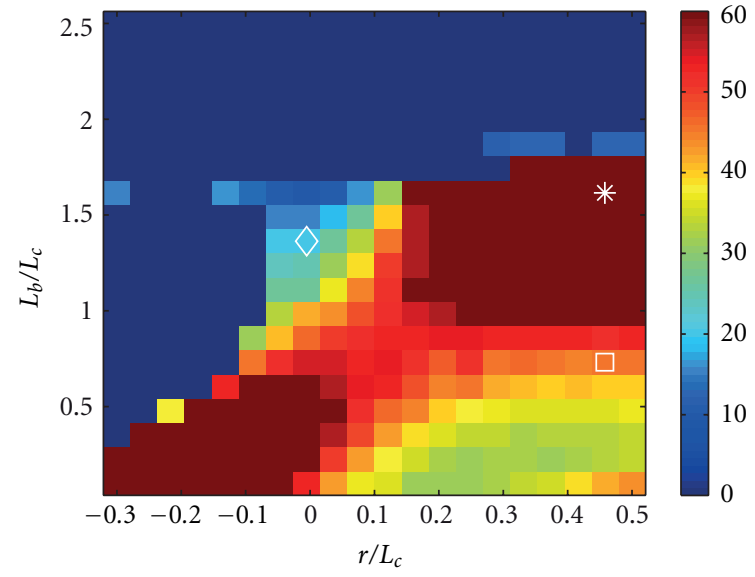

(a)

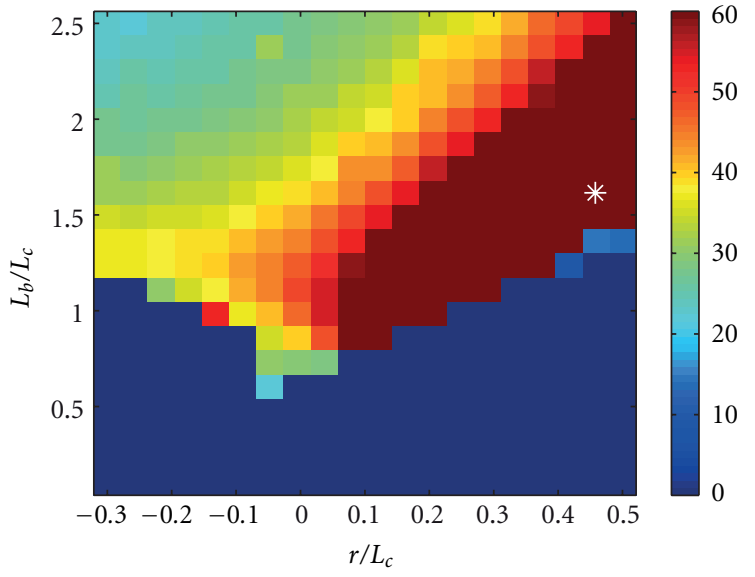

(b)

FIGURE 14: "Double striking propensity" D plotted in the "clappering plane," for (a) ringing right and (b) ringing wrong, calculated using $R=0.2$. Colour scale is truncated at 60 for comparison with later plots. The bell motion was initialised at angle $\theta=171^{\circ}$. White symbols indicate the positions of the bells from Figure 9.

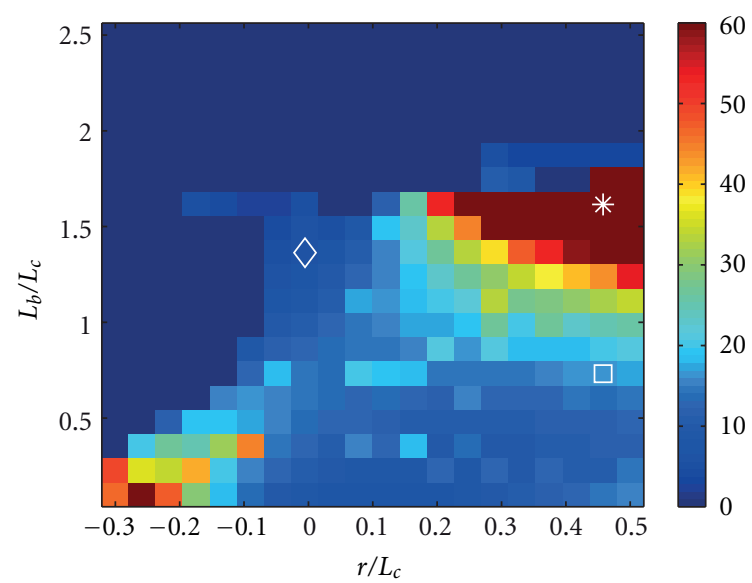

(a)

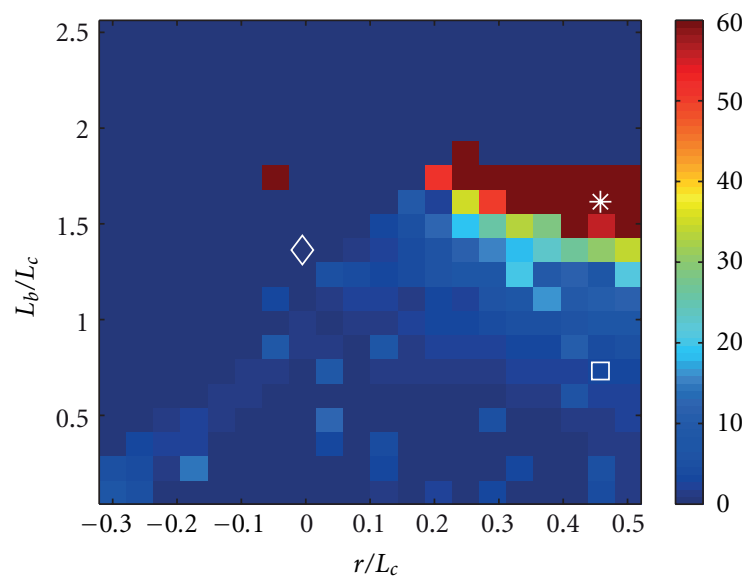

(b)

FIGURE 15: "Double striking propensity" $D$ plotted in the "clappering plane" for bells ringing right, calculated using (a) $R=0.1$ and (b) $R=0.05$. Colour scale matches Figure 14. The bell motion was initialised at angle $\theta=171^{\circ}$. White symbols indicate the positions of the bells from Figure 9.

enough information to evaluate $D$, and it can be concluded that for given values of $\phi_{\max }$ and $R$, the variation of $D$ between different bell configurations should be captured in a clappering chart.

An example is shown in Figure 14, computed using the rather high value $R=0.2$, and it shows that virtually all ringable bells would give a clearly audible double strike. The scale has been truncated at $D=60$ for clarity in later plots; actual values ranged much higher. Recalling that $D=10$ roughly marks the level at which all listeners agreed that a sound was a double strike. Fortunately, the measurements from Section 3.2 indicate that in reality the value of $R$ is likely to be much lower than 0.2 . Figure 15 shows clappering charts for bells "ringing right" for the cases $R=0.1$ and $R=0.05$. As $R$ reduces, the double-strike propensity drops, and with the value 0.05 (which is close to the lowest estimate based on the measurements), only a small region of the chart shows $D>1$. The corresponding plots for ringing wrong, not reproduced here, show a similar pattern, contracting towards the region showing the highest values in Figure 14(b). Interestingly, the laboratory bell falls near this residual region of both charts, so it is not surprising that this bell did indeed tend to produce an audible double strike. As was seen in Figure 2, the laboratory bell also tended to show a longer time interval between initial bounces on one stroke compared to the other; this can probably be attributed to asymmetric behaviour of friction at the clapper pivot, and it produced the effect that the double strike was much more evident on one stroke than the other, as would be predicted from the results here.

4.4. Ringing Up and Ringing Down. All the charts shown so far apply to normal ringing, when the bell turns almost 


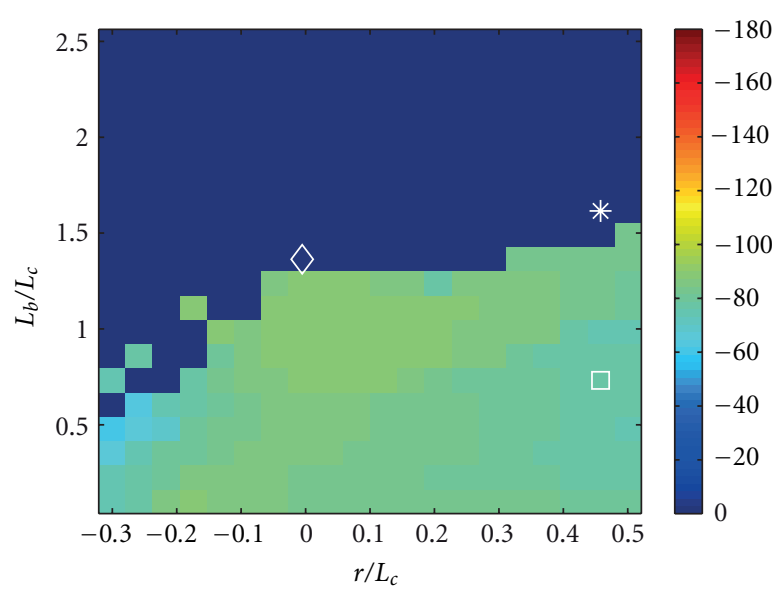

(a)

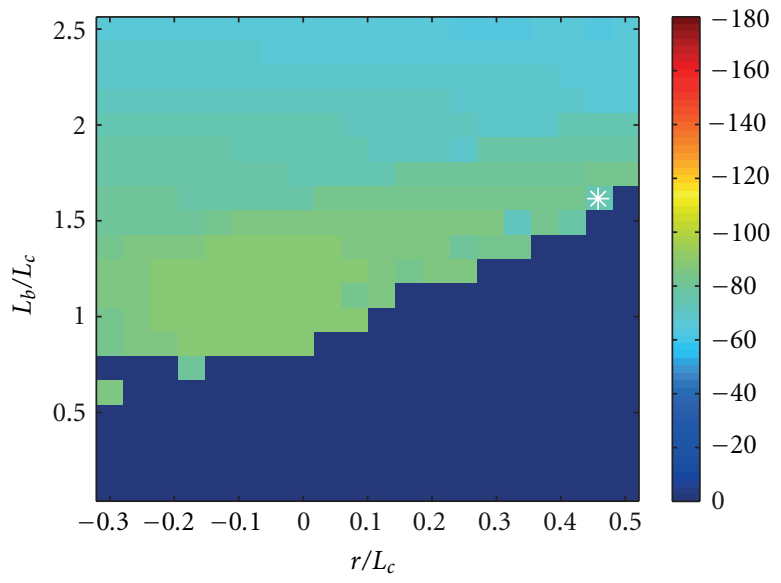

(b)

FIGURE 16: Regions in the "clappering plane" for which a bell can ring (a) right and (b) wrong, for the case in which the bell motion was initialised at angle $\theta=90^{\circ}$ so that the bell swings through a half circle from horizontal to horizontal. Colour shading indicates the bell angle $\theta$ in degrees at which the first contact occurs between clapper and bell, with a scale matching Figure 10. White symbols indicate the positions of the bells from Figure 9 .

a full circle on each stroke. However, for safety, bells are often left in the "down" position when ringing is not taking place, and therefore bells go through a process of being "rung up" and "rung down" at the start and finish of a session. At intermediate stages in this process, the amplitude of bell swing is less than the full circle. To illustrate what happens, clappering charts for the halfway stage, when the bell swings through a half circle starting and finishing horizontal, are shown in Figure 16. These charts, colour shaded to show the angle at first strike, are to be compared with those of Figure 10. Naturally, the colour scale shows that the strike point of the bell never goes beyond $90^{\circ}$.

The regions of the plane in which ringing right and ringing wrong are possible have changed significantly in Figure 16 compared to Figure 10. The upper boundary of the "right" region has moved down, while the "wrong" region has extended into some parts of the plane previously inaccessible. Additional simulations, not shown, using intermediate starting angles reveal that the upper boundary of the "ringing right" region does not begin to move downwards significantly until the initial angle reduces to approximately $130^{\circ}$. The adjustments made in the course of normal changeringing are over a rather small range of angles centred around a value much higher than $130^{\circ}$. This suggests that the problems associated with inadvertently crossing this boundary are not something that change-ringers need to worry about.

This chart shows that it is perfectly possible to hang a bell in such a way that it can be rung right once it is fully rung up, but which is not capable of ringing right at the halfway point shown in this plot. Indeed, the laboratory bell falls into this category. This means that it can be tricky to ring this bell up so that the clapper is on the right side. It also means that if the bell is ringing right and it is then allowed to ring down, at some stage the clapper will do something unexpected and switch over to the other side of the bell so that it rings wrong for the final stages of the ringing down. The laboratory bell did indeed behave in this way. The data in Table 1 shows that the old tenor bell of Great St Mary's was similarly situated, near the upper limit for ringing right, and this bell was known to be somewhat challenging to ring up right.

\section{Conclusions}

In this paper, experimental and theoretical analyses have been combined to reveal some of the complicated dynamics involved in the ringing of church bells in the "English manner." Some unexpected details were revealed by both kinds of analysis. The experimental results show that all church bells, when rung full-circle, produce multiple impacts between the clapper and the bell on every stroke. The clapper may continue to bounce for a second or more, and during that time, the bell continues to vibrate with a decay rate more or less the same as would have occurred following a single, clean impact. However, once the clapper comes to rest against the side of the bell, the vibration is damped out very quickly. The most likely explanation of this is frictional dissipation of energy; the vibration of the bell involves some tangential motion of the surface, and this causes rubbing against the clapper. That rubbing, at the end of every stroke of the bell, is probably also the main cause of the wear scar that always builds up at the two striking points on the bell's surface.

The first stage of theoretical modelling was to establish the governing equations for the bell and clapper. It was shown that under reasonable approximations, these equations, when applied to the phase of motion involving free flight of the bell and clapper, depend on the properties of bell and clapper only via two dimensionless parameters. These parameters are constructed from three lengths: the offset distance $r$ of the pivot axis of the clapper below that of the bell (which could possibly be negative), and the lengths $L_{b}$ and 
$L_{c}$ of the "equivalent simple pendulums" that would swing with the same period as the bell and clapper, respectively. These lengths can be easily deduced from measurements of the small-amplitude swing periods of the bell and clapper, via (9). The two governing parameters are then the ratios $L_{b} / L_{c}$ and $r / L_{c}$. Computer simulation has been used to produce design charts in the plane of these two parameters, called here "clappering charts." These charts show whether a given bell can ring "right," "wrong," or both. They also show the angle of the bell at which the first strike occurs and the relative velocity of the clapper at that moment, which influences the loudness of the bell.

The charts also give an indication of whether a particular bell is likely to produce the audible effect of a double strike. It has already been noted that, in reality, bells always produce multiple strikes. But whether we perceive the sound as a double strike is a different question, determined by psychoacoustical considerations. A simple listening test was carried out with experienced ringers. This led to a parametric description of when a double strike should be heard, and this in turn enabled the computation of a relevant clappering chart.

In order to compute this last chart, two additional dimensionless parameters come into play: the limiting clapper angle $\phi_{\max }$ and, more important, the coefficient of restitution $R$. Measurements on a range of bells show that the effective coefficient of restitution for the first impact of the clapper against the bell always has a very small value; as little as $5 \%$ of the kinetic energy of the clapper motion is recovered in the rebound. Subsequent bounces, however, show much less energy loss. It is suggested that the initial energy loss is associated with setting the bell into vibration, whereas for later bounces, the clapper encounters a bell that is already vibrating.

A simple energy-based argument relating to the excitation of vibration by a rebounding mass shows that there is, at least approximately, a connection between the mass ratio of bell to clapper and the number of overtones of bell vibration that are strongly excited. A heavier clapper can only excite rather few bell modes, while a lighter one can excite more. This argument has implications beyond the particular application to bell ringing, for example to the bandwidth that can be excited when using an impulse hammer to measure the vibration response of a structure.

In summary, it is suggested that the pair of dimensionless parameters could usefully be measured for any ring of bells, especially where there is perceived to be a problem with the sound or handling. Plotting the location of the bells in the clappering charts should reveal general aspects of behaviour, such as loudness, propensity to ring right/wrong, and the likelihood of double striking being heard. Where there is a problem in any of these areas, the charts show immediately what kind of change is necessary to ameliorate it. The plot would also reveal if the bells are neatly clustered so as to have similar handling properties, or whether perhaps one bell stands apart from the others, in which case one might expect it to present some kind of problem for the ringer. As an aside, there may be other issues arising from the fact that bells for change-ringing are, inevitably, in sets. Choices have to be made by bell-founders and bell-hangers about weights and hanging details; it might be interesting to investigate what factors are important beyond the values of the two dimensionless parameters, and whether there is a rational basis for traditional practices.

Fortuitously, the small bell studied in the laboratory exhibits several of the possible bell-hanging problems. It is in the region of the charts where it is possible to ring both right and wrong but sufficiently near the upper limit of the "right" region that during the process of ringing up or down, it ceases to be possible for the bell to be rung right. Being near this upper limit automatically makes it a rather quiet bell, because that limit is determined by the condition that the clapper only just manages to hit the bell. To cap it all, this bell is placed in the particular region of the diagram that shows the worst propensity for double striking. All these predictions were borne out in practice; this bell could serve as a model of bad practice in bell-hanging!

Finally, it may be useful to mention a few issues concerning bells and their accessories that might be of significance but that have not been included in the modelling presented here. No account has been taken of anything to do with mechanical friction in the bearings, or with the rope and the forces applied by the ringer. There may, for example, be significant issues concerned with the weight and mechanical properties of the rope, details arising from the interaction between rope and pulley, and the detailed design of the wheel and headstock to go with a bell of a given size that might form the subject of future work.

\section{Acknowledgments}

The authors thank the many Cambridge ringers who gave assistance during the project and contributed to discussions, particularly Roger Palmer and Tom Ridgman for the loan and setup of the small bell used for laboratory experiments. Brian Moore is thanked for advice on psychoacoustical testing.

\section{References}

[1] N. H. Fletcher and T. D. Rossing, The Physics of Musical Instruments, Springer, 1991.

[2] W. G. Wilson, Change Ringing, Faber, London, UK, 1965.

[3] F. H. King, J. W. McClenahan, and B. D. Threlfall, "The clappering of a bell," The Ringing World, vol. 61, no. 2846, p. $744,1965$.

[4] C. H. Hodges, J. Power, and J. Woodhouse, "The use of the sonogram in structural acoustics and an application to the vibrations of cylindrical shells," Journal of Sound and Vibration, vol. 101, no. 2, pp. 203-218, 1985.

[5] K. L. Johnson, Contact Mechanics, Cambridge University Press, 1987.

[6] N. H. Fletcher, W. T. McGee, and A. Z. Tarnopolsky, "Bell clapper impact dynamics and the voicing of a carillon," Journal of the Acoustical Society of America, vol. 111, no. 3, pp. 14371444, 2002.

[7] J. Klemenc, A. Rupp, and M. Fajdiga, "Dynamics of a clapperto-bell impact," International Journal of Impact Engineering, vol. 44, pp. 29-39, 2012. 
[8] J. W. S. Rayleigh, Theory of Sound, Volume 1, Dover Publications, New York, NY, USA, 1945.

[9] W. J. Stronge, Impact Mechanics, Cambridge University Press, 2004.

[10] W. J. Stronge, "Rigid body collisions with friction," Proceedings of the Royal Society A, vol. 431, pp. 169-181, 1990.

[11] D. J. Ewins, Modal Testing: Theory, Practice and Application, Research Studies Press, 2000.

[12] B. C. J. Moore, An Introduction to the Psychology of Hearing, Elsevier Academic Press, 2004. 

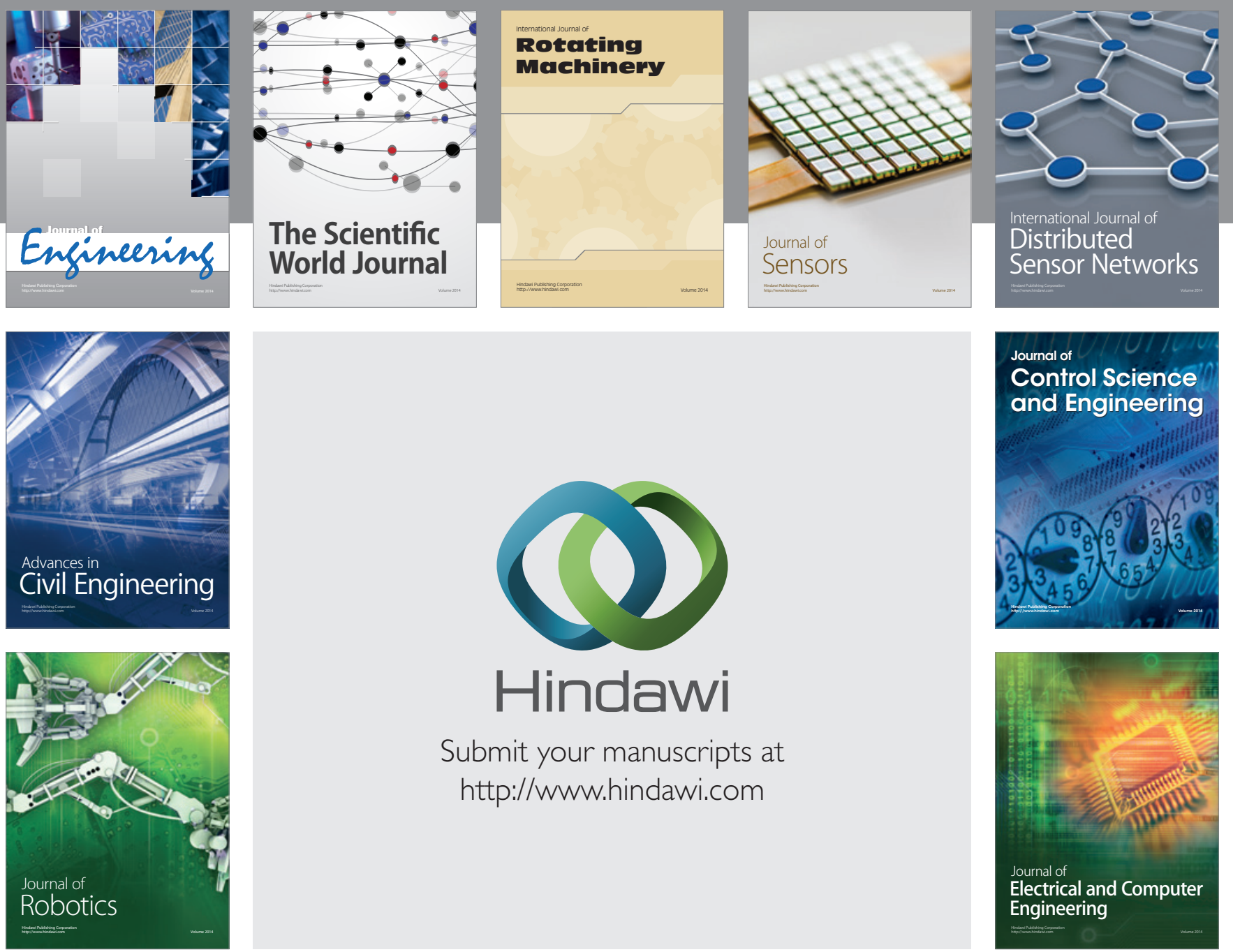

Submit your manuscripts at

http://www.hindawi.com
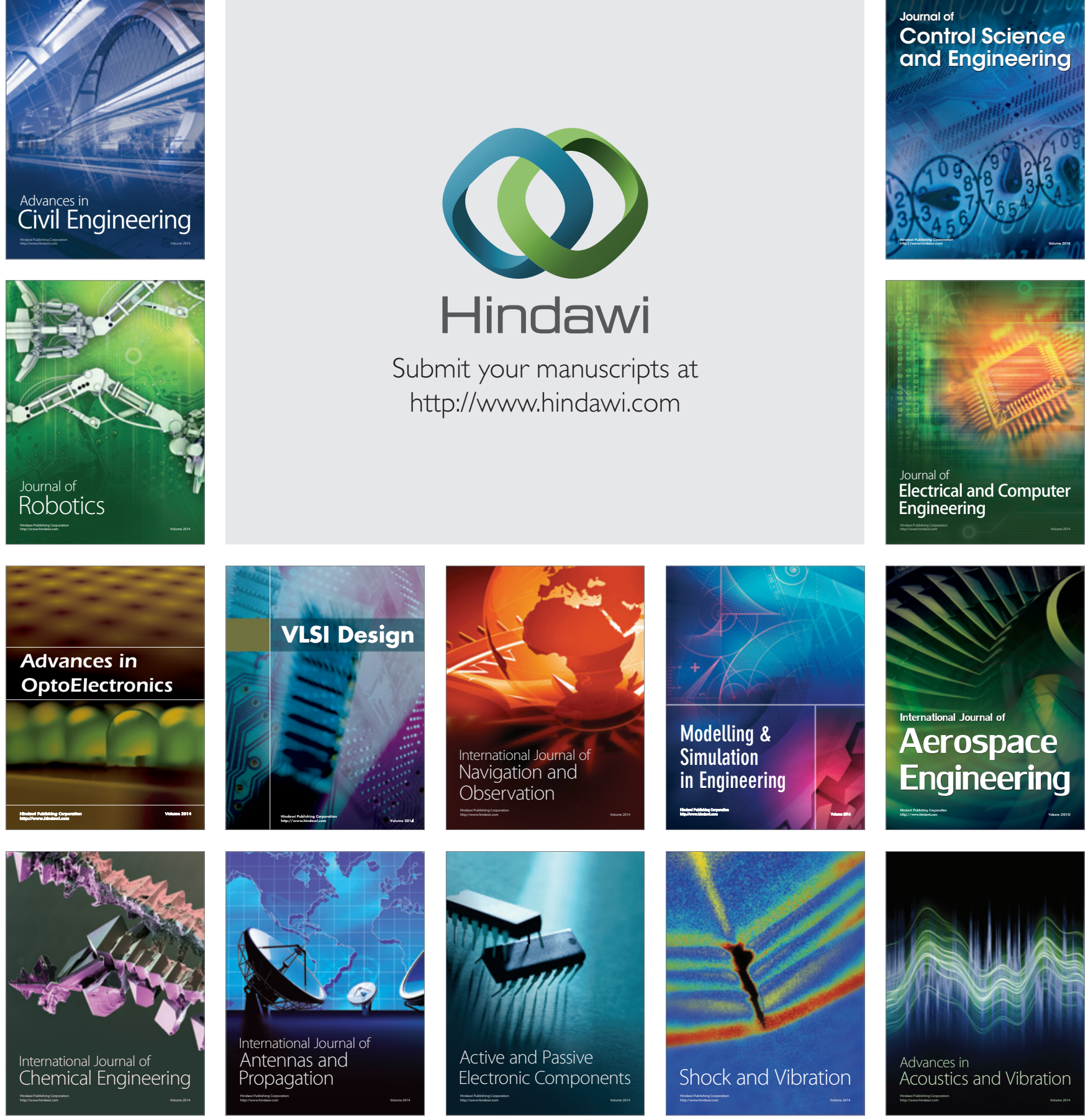